\title{
Dynamics of a two-dimensional system of rational difference equations of Leslie-Gower type
}

\author{
S Kalabušić ${ }^{1}$, MRS Kulenović ${ }^{*}$ and E Pilav ${ }^{1}$
}

\author{
* Correspondence: \\ mkulenovic@mail.uri.edu \\ ${ }^{2}$ Department of Mathematics, \\ University of Rhode Island, \\ Kingston, RI 02881-0816, USA \\ Full list of author information is \\ available at the end of the article
}

\begin{abstract}
We investigate global dynamics of the following systems of difference equations

$$
\left\{\begin{array}{l}
x_{n+1}=\frac{\alpha_{1}+\beta_{1} x_{n}}{A_{1}+y_{n}} \\
y_{n+1}=\frac{\gamma_{2} y_{n}}{A_{2}+B_{2} x_{n}+y_{n}}
\end{array}, \quad n=0,1,2, \ldots\right.
$$

where the parameters $\alpha_{1}, \beta_{1}, A_{1}, \gamma_{2}, A_{2}, B_{2}$ are positive numbers, and the initial conditions $x_{0}$ and $y_{0}$ are arbitrary nonnegative numbers. We show that this system has rich dynamics which depends on the region of parametric space. We show that the basins of attractions of different locally asymptotically stable equilibrium points or non-hyperbolic equilibrium points are separated by the global stable manifolds of either saddle points or non-hyperbolic equilibrium points. We give examples of a globally attractive non-hyperbolic equilibrium point and a semi-stable non-hyperbolic equilibrium point. We also give an example of two local attractors with precisely determined basins of attraction. Finally, in some regions of parameters, we give an explicit formula for the global stable manifold.

Mathematics Subject Classification (2000)

Primary: 39A10, 39A11 Secondary: 37E99, 37D10
\end{abstract}

Keywords: Basin of attraction, Competitive map, Global stable manifold, Monotonicity, Period-two solution

\section{Introduction}

In this paper, we study the global dynamics of the following rational system of difference equations

$$
\left\{\begin{array}{l}
x_{n+1}=\frac{\alpha_{1}+\beta_{1} x_{n}}{A_{1}+y_{n}} \\
y_{n+1}=\frac{\gamma_{2} y_{n}}{A_{2}+B_{2} x_{n}+y_{n}}
\end{array}, \quad n=0,1,2, \ldots\right.
$$

where the parameters $\alpha_{1}, \beta_{1}, A_{1}, \gamma_{2}, A_{2}, B_{2}$ are positive numbers and initial conditions $x_{0}$ and $y_{0}$ are arbitrary nonnegative numbers.

System (1) was mentioned in [1] as one of three systems of Open Problem 3, which asked for a description of the global dynamics of some rational systems of difference equations. In notation used to label systems of linear fractional difference equations 
used in [1], System (1) is referred to as $(29,38)$. This system is dual to the system where the roles of $x_{n}$ and $y_{n}$ are interchanged, which is labeled as $(29,38)$ in [1], and so all results proven here extend to the latter system. In this paper, we provide a precise description of the global dynamics of the System (1). We show that System (1) may have between zero and three equilibrium points, which may have different local character. If System (1) has one equilibrium point, then this point is either locally asymptotically stable or saddle point or non-hyperbolic equilibrium point. If System (1) has two equilibrium points, then they are either locally asymptotically stable and nonhyperbolic, or locally asymptotically stable and saddle point. If System (1) has three equilibrium points, then two of equilibrium points are locally asymptotically stable and the third point, which is between these two points in southeast ordering defined below, is a saddle point. The major problem for global dynamics of the System (1) is determining the basins of attraction of different equilibrium points. The difficulty in analyzing the behavior of all solutions of the System (1) lies in the fact that there are many regions of parameters where this system possesses different equilibrium points with different local character and that in several cases, the equilibrium point is nonhyperbolic. However, all these cases can be handled by using recent results from [2].

System (1) is a competitive system, and our results are based on recent results about competitive systems in the plane, see [2,3]. System (1) can be used as a mathematical model for competition in population dynamics. In fact, second equation in (1) is of Leslie-Gower type, and first equation can be considered to be of Leslie-Gower type with stocking which is represented with the term $\alpha_{1}$, see [4-6].

In the next section, we present some general results about competitive systems in the plane. Section 3 contains some basic facts such as the non-existence of period-two solution of System (1). Section 4 analyzes local stability which is fairly complicated for this system. Finally, Section 5 gives global dynamics for all values of parameters.

\section{Preliminaries}

A first-order system of difference equations

$$
\left\{\begin{array}{l}
x_{n+1}=f\left(x_{n}, y_{n}\right) \\
y_{n+1}=g\left(x_{n}, y_{n}\right)
\end{array}, \quad n=0,1,2, \ldots\right.
$$

where $\mathcal{S} \subset \mathbb{R}^{2},(f, g): \mathcal{S} \rightarrow \mathcal{S}, f, g$ are continuous functions is competitive if $f(x, y)$ is non-decreasing in $x$ and non-increasing in $y$, and $g(x, y)$ is non-increasing in $x$ and non-decreasing in $y$. If both $f$ and $g$ are non-decreasing in $x$ and $y$, the System (2) is cooperative. Competitive and cooperative maps are defined similarly. Strongly competitive systems of difference equations or strongly competitive maps are those for which the functions $f$ and $g$ are coordinate-wise strictly monotone.

Competitive and cooperative systems have been investigated by many authors, see [2,3,5-19]. Special attention to discrete competitive and cooperative systems in the plane was given in $[2,3,5-7,10,12,17,20]$. One of the reasons for paying special attention to two-dimensional discrete competitive and cooperative systems is their applicability and the fact that many examples of mathematical models in biology and economy which involve competition or cooperation are models which involve two species. Another reason is that the theory of two-dimensional discrete competitive and cooperative systems is very well developed, unlike such theory for three and higher 
dimensional systems. Part of the reason for this situation is de Mottoni and Schiaffino theorem given below, which provides relatively simple scenarios for possible behavior of many two-dimensional discrete competitive and cooperative systems. However, this does not mean that one cannot encounter chaos in such systems as has been shown by Smith, see [17].

If $\mathbf{v}=(u, v) \in \mathbb{R}^{2}$, we denote with $\mathcal{Q}_{1}(\mathrm{v}), \ell \in\{1,2,3,4\}$, the four quadrants in $\mathbb{R}^{2}$ relative to v, i.e., $\mathcal{Q}_{1}(\mathrm{v})=\left\{(x, y) \mathbb{R}^{2}: x \geq u, y \geq v\right\}, \mathcal{Q}_{2}(\mathrm{v})=\left\{(x, y) \in \mathbb{R}^{2}: x \leq u, y \geq v\right\}$, and so on. Define the South-East partial order $\preccurlyeq_{s e}$ on $\mathbb{R}^{2}$ by $(x, y) \preccurlyeq_{s e}(s, t)$ if and only if $x \leq s$ and $y \geq t$. Similarly, we define the North-East partial order $\preccurlyeq_{n e}$ on $\mathbb{R}^{2}$ by $(x, y)$ $\preccurlyeq_{n e}(s, t)$ if and only if $x \leq s$ and $y \leq t$. For $\mathcal{A} \subset \mathbb{R}^{2}$ and $\mathrm{x} \in \mathbb{R}^{2}$, define the distance from $\mathrm{x}$ to $\mathcal{A}$ as $\operatorname{dist}(\mathrm{x}, \mathcal{A})=\inf \{|| \mathrm{x}-\mathrm{y} \|: \mathrm{y} \in \mathcal{A}\}$. By int $\mathcal{A}$, we denote the interior of a set $\mathcal{A}$.

It is easy to show that a map $F$ is competitive if it is non-decreasing with respect to the South-East partial order, that is, if the following holds:

$$
\left(\begin{array}{l}
x^{1} \\
y^{1}
\end{array}\right) \preccurlyeq_{s e}\left(\begin{array}{l}
x^{2} \\
y^{2}
\end{array}\right) \Rightarrow F\left(\begin{array}{l}
x^{1} \\
y^{1}
\end{array}\right) \preccurlyeq_{s e} F\left(\begin{array}{l}
x^{2} \\
y^{2}
\end{array}\right) \text {. }
$$

For standard definitions of attracting fixed point, saddle point, stable manifold, and related notions see [11].

We now state three results for competitive maps in the plane. The following definition is from [17].

Definition 1 Let $\mathcal{S}$ be a nonempty subset of $\mathbb{R}^{2}$. A competitive map $T: \mathcal{S} \rightarrow$ Sis said to satisfy condition $(O+)$ if for every $x, y$ in $\mathcal{S}, T(x) \preccurlyeq_{n e} T(y)$ implies $x \preccurlyeq_{n e} y$, and $T$ is said to satisfy condition (O-) if for every $x, y$ in $\mathcal{S}, T(x) \preccurlyeq_{n e} T(y)$ implies $y \leqslant_{n e} x$.

The following theorem was proved by de Mottoni and Schiaffino [20] for the Poincaré map of a periodic competitive Lotka-Volterra system of differential equations. Smith $[14,15]$ generalized the proof to competitive and cooperative maps.

Theorem 1 Let $\mathcal{S}$ be a nonempty subset of $\mathbb{R}^{2}$. If $T$ is a competitive map for which ( $O$ $+)$ holds then for all $x \in \mathcal{S},\left\{T^{n}(x)\right\}$ is eventually componentwise monotone. If the orbit of $x$ has compact closure, then it converges to a fixed point of T. If instead (O-) holds, then for all $x \in \mathcal{S},\left\{T^{2 n}(x)\right\}$ is eventually componentwise monotone. If the orbit of $x$ has compact closure in $\mathcal{S}$, then its omega limit set is either a period-two orbit or a fixed point.

The following result is from [17], with the domain of the map specialized to be the cartesian product of intervals of real numbers. It gives a sufficient condition for conditions $(\mathrm{O}+)$ and $(\mathrm{O}-)$.

Theorem 2 Let $\mathscr{R} \subset \mathbb{R}^{2}$ be the cartesian product of two intervals in $\mathbb{R}$. Let $T: \mathscr{R} \rightarrow$ $\mathscr{R}$ be a $C^{1}$ competitive map. If $T$ is injective and $\operatorname{det} J_{T}(x)>0$ for all $x \in \mathscr{R}$ then $T$ satisfies $(O+)$. If $T$ is injective and $\operatorname{det} J_{T}(x)<0$ for all $x \in \mathscr{R}$ then $T$ satisfies (O-).

The following result is a direct consequence of the Trichotomy Theorem of Dancer and Hess, see [3] and [21] and is helpful for determining the basins of attraction of the equilibrium points.

Corollary 1 If the nonnegative cone of $\leqslant$ is a generalized quadrant in $\mathbb{R}^{n}$, and if $T$ has no fixed points in $\llbracket u_{1}, u_{2} \rrbracket$ other than $u_{1}$ and $u_{2}$, then the interior of $\llbracket u_{1}, u_{2} \rrbracket$ is either a subset of the basin of attraction of $u_{1}$ or a subset of the basin of attraction of $u_{2}$. 
Next result is well-known global attractivity result that holds in partially ordered Banach spaces as well, see [21].

Theorem 3 Let $T$ be a monotone map on a closed and bounded rectangular region $\mathscr{R}$ $\subset \mathbb{R}^{2}$. Suppose that $T$ has a unique fixed point $\overline{\mathbf{e}}$ in $\mathscr{R}$. Then $\overline{\mathbf{e}}$ is a global attractor of $T$ on $\mathscr{R}$.

The following theorems were proved by Kulenović and Merino [2] for competitive systems in the plane, when one of the eigenvalues of the linearized system at an equilibrium (hyperbolic or non-hyperbolic) is by absolute value smaller than 1 while the other has an arbitrary value. These results are useful for determining basins of attraction of fixed points of competitive maps.

Theorem 4 Let $T$ be a competitive map on a rectangular region $\mathscr{R} \subset \mathbb{R}^{2}$. Let $\overline{\mathrm{x}} \in \mathcal{R}$ be a fixed point of $T$ such that $\Delta:=\mathscr{R} \cap$ int $\left(\mathcal{Q}_{1}(\overline{\mathrm{x}}) \cup \mathcal{Q}_{3}(\overline{\mathrm{x}})\right)$ is nonempty (i.e., $\overline{\mathrm{x}}$ is not the NW or SE vertex of $\mathscr{R})$, and $T$ is strongly competitive on $\Delta$. Suppose that the following statements are true.

a. The map $T$ has a $C^{1}$ extension to a neighborhood of $\overline{\mathrm{x}}$.

b. The Jacobian $J_{T}(\overline{\mathrm{x}})$ of $T$ at $\overline{\mathrm{x}}$ has real eigenvalues $\lambda$, $\mu$ such that $0<|\lambda|<\mu$, where $|\lambda|<1$, and the eigenspace $E^{\lambda}$ associated with $\lambda$ is not a coordinate axis.

Then there exists a curve $\mathcal{C} \subset \mathscr{R}$ through $\overline{\mathrm{x}}$ that is invariant and a subset of the basin of attraction of $\overline{\mathrm{x}}$, such that $\mathcal{C}$ is tangential to the eigenspace $E^{\lambda}$ at $\overline{\mathrm{X}}$, and $\mathcal{C}$ is the graph of a strictly increasing continuous function of the first coordinate on an interval. Any endpoints of $\mathcal{C}$ in the interior of $\mathscr{R}$ are either fixed points or minimal period-two points. In the latter case, the set of endpoints of $\mathcal{C}$ is a minimal period-two orbit of $T$.

The situation where the endpoints of $\mathcal{C}$ are boundary points of $\mathscr{R}$ is of interest. The following result gives a sufficient condition for this case.

Theorem 5 For the curve $\mathcal{C}$ of Theorem 4 to have endpoints in $\partial \mathscr{R}$, it is sufficient that at least one of the following conditions is satisfied.

i. The map $T$ has no fixed points nor periodic points of minimal period-two in $\Delta$.

ii. The map $T$ has no fixed points in $\Delta$, det $J_{T}(\overline{\mathrm{x}})>0$, and $T(\overline{\mathrm{x}})=\mathrm{xhas}$ no solutions $\mathrm{x} \in \Delta$.

iii. The map $T$ has no points of minimal period-two in $\Delta$, det $J_{T}(\bar{x})<0$, and $T(\mathrm{x})=\overline{\mathrm{x}}$ has no solutions $\mathrm{x} \in \Delta$.

The next result is useful for determining basins of attraction of fixed points of competitive maps.

Theorem 6 (A) Assume the hypotheses of Theorem 4, and let $\mathcal{C}$ be the curve whose existence is guaranteed by Theorem 4. If the endpoints of $\mathcal{C}$ belong to $\partial \mathscr{R}$, then $\mathcal{C}$ separates $\mathscr{R}$ into two connected components, namely

$$
\mathcal{W}_{-}:=\left\{\mathrm{x} \in \mathcal{R} \backslash \mathcal{C}: \exists \mathrm{y} \in \mathcal{C} \text { with } \mathrm{x} \preccurlyeq s_{s e} \mathrm{y}\right\} \quad \text { and } \quad \mathcal{W}_{+}:=\left\{\mathrm{x} \in \mathcal{R} \backslash \mathcal{C}: \exists \mathrm{y} \in \mathcal{C} \text { with } \mathrm{y} \preccurlyeq{ }_{s e} \mathrm{x}\right\},
$$

such that the following statements are true.

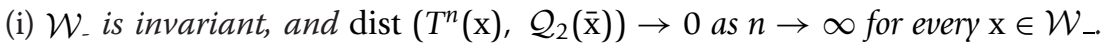

(ii) $\mathcal{W}_{+}$is invariant, and dist $\left(T^{n}(\mathrm{x}), \mathcal{Q}_{4}(\overline{\mathrm{x}})\right) \rightarrow 0$ as $n \rightarrow \infty$ for every $\mathrm{x} \in \mathcal{W}_{+}$. 
(B) If, in addition to the hypotheses of part (A), $\overline{\mathrm{x}}$ is an interior point of $\mathscr{R}$ and $T$ is $\mathrm{C}^{2}$ and strongly competitive in a neighborhood of $\overline{\mathrm{x}}$, then $T$ has no periodic points in the boundary of $\left(\mathcal{Q}_{1}(\overline{\mathrm{x}}) \cup \mathcal{Q}_{3}(\overline{\mathrm{x}})\right)$ except for $\overline{\mathrm{x}}$, and the following statements are true.

(iii) For every $\mathrm{x} \in \mathcal{W}$. there exists $n_{0} \in \mathbb{N}$ such that $T^{n}(\mathrm{x}) \in$ int $\mathcal{Q}_{2}(\overline{\mathrm{x}})$ for $n \geq n_{0}$.

(iv) For every $\mathrm{x} \in \mathcal{W}_{+}$there exists $n_{0} \in \mathbb{N}$ such that $T^{n}(\mathrm{x}) \in \operatorname{int} \mathcal{Q}_{4}(\overline{\mathrm{x}})$ for $n \geq n_{0}$.

If $T$ is a map on a set $\mathscr{R}$ and if $\overline{\mathrm{x}}$ is a fixed point of $T$, the stable set $\mathcal{W}^{s}(\overline{\mathrm{x}})$ of $\overline{\mathrm{x}}$ is the set $\left\{x \in \mathcal{R}: T^{n}(\mathrm{x}) \rightarrow \overline{\mathrm{x}}\right\}$ and unstable set $\mathcal{W}^{u}(\overline{\mathrm{x}})$ of $\overline{\mathrm{x}}$ is the set

$$
\left\{\mathrm{x} \in \mathcal{R} \text { : there exists }\left\{\mathrm{x}_{n}\right\}_{n=-\infty}^{0} \subset \mathcal{R} \text { s.t. } T\left(\mathrm{x}_{n}\right)=\mathrm{x}_{n+1}, \mathrm{x}_{0}=\mathrm{x} \text {, and } \lim _{n \rightarrow-\infty} \mathrm{x}_{n}=\overline{\mathrm{x}}\right\}
$$

When $T$ is non-invertible, the set $\mathcal{W}^{s}(\bar{x})$ may not be connected and made up of infinitely many curves, or $\mathcal{W}^{u}(\bar{x})$ may not be a manifold. The following result gives a description of the stable and unstable sets of a saddle point of a competitive map. If the map is a diffeomorphism on $\mathscr{R}$, the sets $\mathcal{W}^{s}(\bar{x})$ and $\mathcal{W}^{u}(\bar{x})$ are the stable and unstable manifolds of $\overline{\mathrm{x}}$.

Theorem 7 In addition to the hypotheses of part (B) of Theorem 6, suppose that $\mu>1$ and that the eigenspace $E^{\mu}$ associated with $\mu$ is not a coordinate axis. If the curve $\mathcal{C}$ of Theorem 4 has endpoints in $\partial \mathscr{R}$, then $\mathcal{C}$ is the stable set $\mathcal{W}^{s}(\overline{\mathrm{x}})$ of $\overline{\mathrm{x}}$, and the unstable set $\mathcal{W}^{u}(\overline{\mathrm{x}})$ of $\bar{x}$ is a curve in $\mathscr{R}$ that is tangential to $E^{\mu}$ at $\overline{\mathrm{x}}$ and such that it is the graph of a strictly decreasing function of the first coordinate on an interval. Any endpoints of $\mathcal{W}^{u}(\bar{x})$ in $\mathscr{R}$ are fixed points of $T$.

The following result gives information on local dynamics near a fixed point of a map when there exists a characteristic vector whose coordinates have negative product and such that the associated eigenvalue is hyperbolic. This is a well-known result, valid in much more general setting that we include it here for completeness. A point $(x, y)$ is a subsolution if $T(x, y) \preccurlyeq_{s e}(x, y)$, and $(x, y)$ is a supersolution if $(x, y) \preccurlyeq_{s e} T(x, y)$. An order interval $\llbracket(a, b),(c, d) \rrbracket$ is the cartesian product of the two compact intervals $[a, c]$ and $[b, d]$.

Theorem 8 Let $T$ be a competitive map on a rectangular set $\mathscr{R} \subset \mathbb{R}^{2}$ with an isolated fixed point $\overline{\mathrm{x}} \in \mathcal{R}$ such that $\mathcal{R} \cap \operatorname{int}\left(\mathcal{Q}_{2}(\overline{\mathrm{x}}) \cup \mathcal{Q}_{4}(\overline{\mathrm{x}})\right) \neq \emptyset$. Suppose $T$ has a $C^{1}$ extension to a neighborhood of $\overline{\mathrm{x}}$. Let $\mathrm{v}=\left(\mathrm{v}^{(1)}, \mathrm{v}^{(2)}\right) \in \mathbb{R}^{2}$ be an eigenvector of the Jacobian of $T$ at $\overline{\mathrm{X}}$, with associated eigenvalue $\mu \in \mathbb{R}$. If $\mathrm{v}^{(1)} \mathrm{v}^{(2)}<0$, then there exists an order interval $\mathscr{J}$ which is also a relative neighborhood of $\overline{\mathrm{x}}$ such that for every relative neighborhood $\mathcal{U} \subset \mathscr{g}$ of $\overline{\mathrm{x}}$ the following statements are true.

i. If $\mu>1$, then $\mathcal{U} \cap$ int $\mathcal{Q}_{2}(\overline{\mathrm{x}})$ contains a subsolution and $\mathcal{U} \cap$ int $\mathcal{Q}_{4}(\overline{\mathrm{x}})$ contains a supersolution. In this case for every $\mathrm{x} \in \mathcal{I} \cap$ int $\left(\mathcal{Q}_{2}(\overline{\mathrm{x}}) \cup \mathcal{Q}_{4}(\overline{\mathrm{x}})\right)$ there exists $N$ such that $T^{n}(\mathrm{x}) \notin \mathscr{D}$ for $n \geq N$.

ii. If $\mu<1$, then $\mathcal{U} \cap$ int $\mathcal{Q}^{2}(\overline{\mathrm{x}})$ contains a supersolution and $\mathcal{U} \cap$ int $\mathcal{Q}_{4}(\overline{\mathrm{x}})$ contains a subsolution. In this case $T^{n}(\mathrm{x}) \rightarrow \overline{\mathrm{x}}$ for every $\mathrm{x} \in \mathscr{Z}$.

\section{Some basic facts}

In this section, we give some basic facts about the nonexistence of period-two solutions, local injectivity of the map $T$ at the equilibrium point, and boundedness of solutions. See [22] for similar analysis. 


\subsection{Equilibrium points}

The equilibrium points $(\overline{\mathrm{x}}, \bar{y})$ of System (1) satisfy

$$
\bar{x}=\frac{\alpha_{1}+\beta_{1} \bar{x}}{A_{1}+\bar{y}}, \quad \bar{y}=\frac{\gamma_{2} \bar{y}}{A_{2}+B_{2} \bar{x}+\bar{y}} .
$$

Solutions of System (5) are:

(i) $\bar{y}=0, \bar{x}=\frac{\alpha_{1}}{A_{1}-\beta_{1}}, A_{1}>\beta_{1}$, i.e. $E_{1}=\left(\frac{\alpha_{1}}{A_{1}-\beta_{1}}, 0\right)$. Thus, the equilibrium point $E_{1}=\left(\frac{\alpha_{1}}{A_{1}-\beta_{1}}, 0\right)$ exists if $A_{1}>\beta_{1}$.

(ii) If $\bar{\gamma} \neq 0$, then using System (5), we obtain

$$
\bar{y}=\gamma_{2}-A_{2}-B_{2} \bar{x}, \quad \bar{x}^{2} B_{2}-\bar{x}\left(\gamma_{2}+A_{1}-A_{2}-\beta_{1}\right)+\alpha_{1}=0 .
$$

Solutions of System (6) are:

$$
\bar{x}_{3,2}=\frac{\gamma_{2}+A_{1}-A_{2}-\beta_{1} \pm \sqrt{D_{0}}}{2 B_{2}}, \quad \bar{y}_{2,3}=\frac{\gamma_{2}-A_{2}-A_{1}+\beta_{1} \pm \sqrt{D_{0}}}{2},
$$

where $D_{0}=\left(\gamma_{2}-A_{2}+A_{1}-\beta_{1}\right)^{2}-4 B_{2} \alpha_{1}$ which gives a pair of the equilibrium points $E_{2}=\left(\bar{x}_{2}, \bar{y}_{2}\right)$ and $E_{3}=\left(\bar{x}_{3}, \bar{y}_{3}\right)$.

The criteria for the existence of the three equilibrium points are summarized in Table 1.

\subsection{Injectivity}

Lemma 1 Assume that $(\bar{x}, \bar{y})$ is an equilibrium of the map $T$. Then the following holds:

1) If

$$
B_{2}>\frac{A_{2} \beta_{1}}{\alpha_{1}}, \quad A_{1}\left(B_{2} \alpha_{1}-A_{2} \beta_{1}\right) \gamma_{2}-\left(B_{2} \alpha_{1}+\left(A_{1}-A_{2}\right) \beta_{1}\right)\left(A_{1} A_{2}-\beta_{1} A_{2}+B_{2} \alpha_{1}\right)=0,
$$

then $T\left(x, \frac{A_{1} A_{2} \beta_{1}+x A_{1} B_{2} \beta_{1}}{B_{2} \alpha_{1}-A_{2} \beta_{1}}\right)=(\bar{x}, \bar{y})$ for all $x \geq 0$, where

$$
(\bar{x}, \bar{y})=\left(\bar{x}, \frac{A_{1} A_{2} \beta_{1}+\bar{x} A_{1} B_{2} \beta_{1}}{B_{2} \alpha_{1}-A_{2} \beta_{1}}\right)=\left(\frac{B_{2} \alpha_{1}+A_{2} \beta_{1}}{A_{1} B_{2}}, \frac{-A_{2} \beta_{1}^{2}+A_{1} A_{2} \beta_{1}+B_{2} \alpha_{1} \beta_{1}}{B_{2} \alpha_{1}-A_{2} \beta_{1}}\right) .
$$

That is the line

$$
\mathcal{I}=\left\{\left(x, \frac{A_{1} A_{2} \beta_{1}+x A_{1} B_{2} \beta_{1}}{B_{2} \alpha_{1}-A_{2} \beta_{1}}\right): x \geq 0\right\}
$$

is invariant, equilibrium $(\bar{x}, \bar{y}) \in \mathcal{I}$ and for $(\mathrm{x}, \mathrm{y}) \in \mathscr{J}$ the following holds $T(x, y)=(\bar{x}, \bar{y})$, that is every point of this line is mapped to the equilibrium point $(\bar{x}, \bar{y})$.

1.i) If $\left(B_{2} \alpha_{1}-A_{2} \beta_{1}\right)^{2}-A_{1}^{2} B_{2} \alpha_{1}>0$ then $(\bar{x}, \bar{y})=E_{3}$.

1.ii) If $\left(B_{2} \alpha_{1}-A_{2} \beta_{1}\right)^{2}-A_{1}^{2} B_{2} \alpha_{1}<$ then $(\bar{x}, \bar{y})=E_{2}$.

1.iii) If $\left(B_{2} \alpha_{1}-A_{2} \beta_{1}\right)^{2}-A_{1}^{2} B_{2} \alpha_{1}=0$ then $(\bar{x}, \bar{y})=E_{3}=E_{2}$.

2) If 


\begin{tabular}{|c|c|}
\hline$E_{1}$ & $\begin{array}{l}A_{1}>\beta_{1}, A_{2}<\gamma_{2}<A_{1}+A_{2}-\beta_{1}, \frac{\left(A_{1}-\beta_{1}\right)\left(\gamma_{2}-A_{2}\right)}{B_{2}}<\alpha_{1} \leq \frac{\left(A_{1}-A_{2}-\beta_{1}+\gamma_{2}\right)^{2}}{4 B_{2}} \text { or } \\
A_{1}>\beta_{1}, A_{2}>\gamma_{2}, \alpha_{1} \leq \frac{\left(A_{1}-A_{2}-\beta_{1}+\gamma_{2}\right)^{2}}{4 B_{2}}, A_{1}+\gamma_{2} \neq A_{2}+\beta_{1} \text { or } \\
A_{1}>\beta_{1}, A_{2}=\gamma_{2}, \alpha_{1} \leq \frac{\left(A_{1}-A_{2}-\beta_{1}+\gamma_{2}\right)^{2}}{4 B_{2}} \text { or } \\
A_{1}>\beta_{1}, \alpha_{1}>\frac{\left(A_{1}-A_{2}-\beta_{1}+\gamma_{2}\right)^{2}}{4 B_{2}}\end{array}$ \\
\hline$E_{1} \equiv E_{2} \equiv E_{3}$ & $A_{1}>\beta_{1}, A_{1}+A_{2}=\beta_{1}+\gamma_{2}, \alpha_{1}=\frac{\left(A_{1}-\beta_{1}\right)\left(\gamma_{2}-A_{2}\right)}{B_{2}}$ \\
\hline$E_{1} \equiv E_{3}, E_{2}$ & $A_{1}>\beta_{1}, A_{1}+A_{2}<\beta_{1}+\gamma_{2}, A_{2}<\gamma_{2}, \alpha_{1}=\frac{\left(A_{1}-\beta_{1}\right)\left(\gamma_{2}-A_{2}\right)}{B_{2}}$ \\
\hline$E_{1}, E_{2}, E_{3}$ & $A_{1}>\beta_{1}, A_{1}+A_{2}<\beta_{1}+\gamma_{2}, \frac{\left(A_{1}-\beta_{1}\right)\left(\gamma_{2}-A_{2}\right)}{B_{2}}<\alpha_{1}<\frac{\left(A_{1}-A_{2}-\beta_{1}+\gamma_{2}\right)^{2}}{4 B_{2}}$ \\
\hline$E_{1}, E_{2}$ & $A_{1}>\beta_{1}, A_{2}<\gamma_{2}, \alpha_{1}<\frac{\left(A_{1}-\beta_{1}\right)\left(\gamma_{2}-A_{2}\right)}{B_{2}}$ \\
\hline$E_{1} \equiv E_{2}$ & $A_{1}>\beta_{1}, A_{2}<\gamma_{2}<A_{1}+A_{2}-\beta_{1}, \alpha_{1}=\frac{\left(A_{1}-\beta_{1}\right)\left(\gamma_{2}-A_{2}\right)}{B_{2}}$ \\
\hline$E_{1}, E_{2} \equiv E_{3}$ & $A_{1}>\beta_{1}, A_{1}+A_{2}<\beta_{1}+\gamma_{2}, \alpha_{1}=\frac{\left(A_{1}-A_{2}-\beta_{1}+\gamma_{2}\right)^{2}}{4 B_{2}}$ \\
\hline$E_{2}, E_{3}$ & $\begin{array}{l}A_{1}<\beta_{1}, A_{1}+\gamma_{2}>A_{2}+\beta_{1}, \alpha_{1}<\frac{\left(A_{1}-A_{2}-\beta_{1}+\gamma_{2}\right)^{2}}{4 B_{2}} \text { or } \\
A_{1}=\beta_{1}, A_{1}+A_{2}<\beta_{1}+\gamma_{2}, \alpha_{1}<\frac{\left(A_{1}-A_{2}-\beta_{1}+\gamma_{2}\right)^{2}}{4 B_{2}}\end{array}$ \\
\hline$E_{2}=E_{3}$ & $\begin{array}{l}A_{1}<\beta_{1} A_{1}+\gamma_{2}>A_{2}+\beta_{1}, \alpha_{1}=\frac{\left(A_{1}-A_{2}-\beta_{1}+\gamma_{2}\right)^{2}}{4 B_{2}} \text { or } \\
A_{1}=\beta_{1}, A_{1}+A_{2}<\beta_{1}+\gamma_{2}, \alpha_{1}=\frac{\left(A_{1}-A_{2}-\beta_{1}+\gamma_{2}\right)^{2}}{4 B_{2}}\end{array}$ \\
\hline No equilibrium & $\begin{array}{l}A_{1}<\beta_{1}, A_{2}<\gamma_{2}<-A_{1}+A_{2}+\beta_{1}, \alpha_{1} \leq \frac{\left(A_{1}-A_{2}-\beta_{1}+\gamma_{2}\right)^{2}}{4 B_{2}} \text { or } \\
A_{1}<\beta_{1}, A_{2} \geq \gamma_{2}, \alpha_{1} \leq \frac{\left(A_{1}-A_{2}-\beta_{1}+\gamma_{2}\right)^{2}}{4 B_{2}} \text { or } \\
A_{1} \leq \beta_{1}, \alpha_{1}>\frac{\left(A_{1}-A_{2}-\beta_{1}+\gamma_{2}\right)^{2}}{4 B_{2}} \text { or } \\
A_{1}=\beta_{1}, A_{1}+A_{2}>\gamma_{2}+\beta_{1}, \alpha_{1} \leq \frac{\left(A_{1}-A_{2}-\beta_{1}+\gamma_{2}\right)^{2}}{4 B_{2}}\end{array}$ \\
\hline
\end{tabular}

$$
B_{2} \leq \frac{A_{2} \beta_{1}}{\alpha_{1}} \text { or } A_{1}\left(B_{2} \alpha_{1}-A_{2} \beta_{1}\right) \gamma_{2}-\left(B_{2} \alpha_{1}+\left(A_{1}-A_{2}\right) \beta_{1}\right)\left(A_{1} A_{2}-\beta_{1} A_{2}+B_{2} \alpha_{1}\right) \neq 0
$$

then the following holds.

$$
T(x, y)=(\bar{x}, \bar{y}) \Rightarrow(x, y)=(\bar{x}, \bar{y}) .
$$

Proof $T(x, y)=(\bar{x}, \bar{y})$ is equivalent to

$$
\left(\frac{\alpha_{1}+\beta_{1} x}{A_{1}+y}, \frac{\gamma_{2} y}{A_{2}+B 2 x+y}\right)=(\bar{x}, \bar{y}) \text {. }
$$

Since $(\bar{x}, \bar{y})$ is the equilibrium point of the map $T$ then System (9) is equivalent to

$$
\left(\frac{\alpha_{1}+\beta_{1} x}{A_{1}+\gamma}, \frac{\gamma_{2} y}{A_{2}+B 2 x+y}\right)=\left(\frac{\alpha_{1}+\beta_{1} \bar{x}}{A_{1}+\bar{y}}, \frac{\gamma_{2} \bar{y}}{A_{2}+B 2 \bar{x}+\bar{y}}\right) \text {. }
$$

System (10) is equivalent to

$$
\begin{aligned}
& -y \alpha_{1}+\bar{\gamma} \alpha_{1}-y \bar{x} \beta_{1}+x \bar{y} \beta_{1}+x A_{1} \beta_{1}-\bar{x} A_{1} \beta_{1}=0 \\
& y A_{2} \gamma_{2}-\bar{y} A_{2} \gamma_{2}+y \bar{x} B_{2} \gamma_{2}-x \bar{y} B_{2} \gamma_{2}=0 .
\end{aligned}
$$


Equation 11 implies

$$
y=\frac{\bar{y} \alpha_{1}+x \bar{y} \beta_{1}+x A_{1} \beta_{1}-\bar{x} A_{1} \beta_{1}}{\alpha_{1}+\bar{x} \beta_{1}} .
$$

and Equation 12 is equivalent to

$$
(x-\bar{x})\left(-\bar{\gamma} B_{2} \alpha_{1}+\bar{y} A_{2} \beta_{1}+A_{1} A_{2} \beta_{1}+\bar{x} A_{1} B_{2} \beta_{1}\right) \gamma_{2}=0 .
$$

We conclude the following: If $\left(-\bar{y} B_{2} \alpha_{1}+\bar{y} A_{2} \beta_{1}+A_{1} A_{2} \beta_{1}+\bar{x} A_{1} B_{2} \beta_{1}\right) \neq 0$, then $x=\bar{x}$ and $y=\bar{y}$.

On the other hand, if $\left(-\bar{y} B_{2} \alpha_{1}+\bar{y} A_{2} \beta_{1}+A_{1} A_{2} \beta_{1}+\bar{x} A_{1} B_{2} \beta_{1}\right)=0$, since $(\bar{x}, \bar{y})$ is the equilibrium of the map $T$, then

$$
B_{2}>\frac{A_{2} \beta_{1}}{\alpha_{1}}, \bar{y}=-\frac{A_{1}\left(A_{2}+\bar{x} B_{2}\right) \beta_{1}}{A_{2} \beta_{1}-B_{2} \alpha_{1}}
$$

and

$$
(\bar{x}, \bar{y})=\left(\frac{\alpha_{1}+\beta_{1} \bar{x}}{A_{1}+\bar{y}}, \frac{\gamma_{2} \bar{y}}{A_{2}+B 2 \bar{x}+\bar{y}}\right) .
$$

Using these equations, we have

$$
\bar{x}=\frac{B_{2} \alpha_{1}-A_{2} \beta_{1}}{A_{1} B_{2}}, \quad \bar{y}=\frac{\beta_{1}\left(-A_{1} A_{2}+\beta_{1} A_{2}-B_{2} \alpha_{1}\right)}{A_{2} \beta_{1}-B_{2} \alpha_{1}}
$$

and

$$
A_{1}\left(B_{2} \alpha_{1}-A_{2} \beta_{1}\right) \gamma_{2}-\left(B_{2} \alpha_{1}+\left(A_{1}-A_{2}\right) \beta_{1}\right)\left(A_{1} A_{2}-\beta_{1} A_{2}+B_{2} \alpha_{1}\right)=0,
$$

which completes the proof of lemma.

\subsection{Period-two solutions}

In this section, we prove that System (1) has no minimal period-two solutions which will be essential for application of Theorem 4 and Corollary 6 .

Lemma 2 System (1) has no minimal period-two solution.

Proof Period-two solution satisfies $T^{2}(x, y)=(x, y)$, that is

$$
T^{2}(x, y)=\left(\frac{\alpha_{1}+\frac{\beta_{1}\left(\alpha_{1}+x \beta_{1}\right)}{\gamma+A_{1}}}{A_{1}+\frac{\gamma \gamma_{2}}{\gamma+A_{2}+x B_{2}}}, \frac{\gamma \gamma_{2}^{2}}{\frac{\left(\gamma+A_{2}+x B_{2}\right)\left(\left(\gamma+A_{1}\right) A_{2}+B_{2}\left(\alpha_{1}+x \beta_{1}\right)\right)}{\gamma+A_{1}}+\gamma \gamma_{2}}\right)=(x, y) .
$$

This is equivalent to

$$
-\frac{\left(y+A_{2}+x B_{2}\right)\left(-x \beta_{1}^{2}-\alpha_{1} \beta_{1}+\left(y+A_{1}\right)\left(x A_{1}-\alpha_{1}\right)\right)+x y\left(y+A_{1}\right) \gamma_{2}}{\left(y+A_{1}\right)\left(A_{1}\left(\gamma+A_{2}+x B_{2}\right)+y \gamma_{2}\right)}=0
$$

and

$$
\frac{y\left(\left(y+A_{1}\right) \gamma_{2}^{2}-y\left(y+A_{1}\right) \gamma_{2}+\left(-y-A_{2}-x B_{2}\right)\left(\left(y+A_{1}\right) A_{2}+B_{2}\left(\alpha_{1}+x \beta_{1}\right)\right)\right)}{\left(y+A_{2}+x B_{2}\right)\left(\left(y+A_{1}\right) A_{2}+B_{2}\left(\alpha_{1}+x \beta_{1}\right)\right)+y\left(y+A_{1}\right) \gamma_{2}}=0,
$$

which is equivalent to

$$
\left(y+A_{2}+x B_{2}\right)\left(-x \beta_{1}^{2}-\alpha_{1} \beta_{1}+\left(y+A_{1}\right)\left(x A_{1}-\alpha_{1}\right)\right)+x y\left(y+A_{1}\right) \gamma_{2}=0
$$




$$
y\left(\left(y+A_{1}\right) \gamma_{2}^{2}-\gamma\left(\gamma+A_{1}\right) \gamma_{2}+\left(-y-A_{2}-x B_{2}\right)\left(\left(y+A_{1}\right) A_{2}+B_{2}\left(\alpha_{1}+x \beta_{1}\right)\right)\right)=0
$$

If $y=0$, we substitute in (15) to obtain the first fixed point, that is $x=\frac{\alpha_{1}}{A_{1}-\beta_{1}} \mathrm{i}$ $x=-\frac{A_{2}}{B_{2}}$. Assume

$$
\left(y+A_{1}\right) \gamma_{2}^{2}-\gamma\left(y+A_{1}\right) \gamma_{2}+\left(-y-A_{2}-x B_{2}\right)\left(\left(y+A_{1}\right) A_{2}+B_{2}\left(\alpha_{1}+x \beta_{1}\right)\right)=0 .
$$

From (17) we calculate $x^{2}$. We have

$$
\begin{gathered}
x^{2}=-\frac{\left(y+A_{1}\right) A_{2}^{2}+\left(y^{2}+A_{1}\left(y+x B_{2}\right)+B_{2}\left(\alpha_{1}+x\left(y+\beta_{1}\right)\right)\right) A_{2}}{B_{2}^{2} \beta_{1}} \\
-\frac{x B_{2}^{2} \alpha_{1}+\gamma B_{2}\left(\alpha_{1}+x \beta_{1}\right)+\left(y+A_{1}\right)\left(y-\gamma_{2}\right) \gamma_{2}}{B_{2}^{2} \beta_{1}} .
\end{gathered}
$$

Put (18) into (15), we have that (15) is equivalent to

$$
y+A_{1}=0
$$

or

$$
\begin{aligned}
& \left(A_{1}\left(y+A_{1}\right)-\beta_{1}^{2}\right) \gamma_{2}^{2}+y\left(\beta_{1}^{2}+x B_{2} \beta_{1}-A_{1}\left(y+A_{1}\right)\right) \gamma_{2} \\
& \quad+\left(-y-A_{2}-x B_{2}\right)\left(-A_{2} \beta_{1}^{2}+B_{2} \alpha_{1} \beta_{1}+A_{1}\left(\left(y+A_{1}\right) A_{2}+B_{2} \alpha \cdot 1\right)\right)=0
\end{aligned}
$$

If (19) holds, then we obtain a negative solution. Now, assume that (20) holds. We have

$$
\begin{gathered}
x=\frac{\left(A_{1}\left(y+A_{1}\right)-\beta_{1}^{2}\right) \gamma_{2}^{2}-\gamma\left(A_{1}\left(y+A_{1}\right)-\beta_{1}^{2}\right) \gamma_{2}}{B_{2}\left(A_{2} A_{1}^{2}+\left(y A_{2}+B_{2} \alpha_{1}\right) A_{1}-\beta_{1}\left(-B_{2} \alpha_{1}+A_{2} \beta_{1}+y \gamma_{2}\right)\right)} \\
+\frac{\left(-y-A_{2}\right)\left(-A_{2} \beta_{1}^{2}+B_{2} \alpha_{1} \beta_{1}+A_{1}\left(\left(y+A_{1}\right) A_{2}+B_{2} \alpha_{1}\right)\right)}{B_{2}\left(A_{2} A_{1}^{2}+\left(y A_{2}+B_{2} \alpha_{1}\right) A_{1}-\beta_{1}\left(-B_{2} \alpha_{1}+A_{2} \beta_{1}+\gamma \gamma_{2}\right)\right)} .
\end{gathered}
$$

Put (21) into (18), we obtain that (18) is equivalent to

$$
-y^{2}+\left(-A_{1}-A_{2}+\beta_{1}+\gamma_{2}\right) y-B_{2} \alpha_{1}+\beta_{1}\left(A_{2}-\gamma_{2}\right)+A_{1}\left(\gamma_{2}-A_{2}\right)=0
$$

or

$$
\begin{aligned}
- & \left(A_{2}+\gamma_{2}\right)\left(A_{1}^{2}+\left(\beta_{1}-A_{2}\right) A_{1}+\beta_{1} \gamma_{2}\right) \gamma^{2}-\left(A_{1}+\beta_{1}\right)\left(A_{1}-A_{2}+\beta_{1}-\gamma_{2}\right) \\
& \times\left(B_{2} \alpha_{1}+A_{1}\left(A_{2}+\gamma_{2}\right)-\beta_{1}\left(A_{2}+\gamma_{2}\right)\right) y+\left(A_{1}+\beta_{1}\right)^{2} \gamma_{2}\left(B_{2} \alpha_{1}+A_{1}\left(A_{2}+\gamma_{2}\right)-\beta_{1}\left(A_{2}+\gamma_{2}\right)\right)=0 .
\end{aligned}
$$

If (22) holds, we obtain the fixed points. So, we assume that (23) holds. Set

$$
\begin{aligned}
\Delta: & =\left(A_{1}+\beta_{1}\right)^{2}\left(B_{2} \alpha_{1}+\left(A_{1}-\beta_{1}\right)\left(A_{2}+\gamma_{2}\right)\right) \\
& \times\left(\left(B_{2} \alpha_{1}+\left(A_{1}-\beta_{1}\right)\left(A_{2}+\gamma_{2}\right)\right)\left(A_{1}-A_{2}+\beta_{1}-\gamma_{2}\right)^{2}+4 \gamma_{2}\left(A_{2}+\gamma_{2}\right)\left(A_{1}\left(A_{1}-A_{2}+\beta_{1}\right)+\beta_{1} \gamma_{2}\right)\right) .
\end{aligned}
$$

If $\Delta \geq 0$ and $A_{1}\left(A_{1}-A_{2}+\beta_{1}\right)+\beta_{1} \gamma_{2} \neq 0$ hold, we obtain the real solution of the form

$$
\begin{aligned}
& y_{1}=-\frac{\left(\Delta_{1}-\sqrt{\Delta}\right)}{2\left(A_{2}+\gamma_{2}\right)\left(A_{1}\left(A_{1}-A_{2}+\beta_{1}\right)+\beta_{1} \gamma_{2}\right)} \\
& y_{2}=-\frac{\left(\Delta_{1}+\sqrt{\Delta}\right)}{2\left(A_{2}+\gamma_{2}\right)\left(A_{1}\left(A_{1}-A_{2}+\beta_{1}\right)+\beta_{1} \gamma_{2}\right)}
\end{aligned}
$$


where

$$
\Delta_{1}:=\left(A_{1}+\beta_{1}\right)\left(A_{1}-A_{2}+\beta_{1}-\gamma_{2}\right)\left(B_{2} \alpha_{1}+\left(A_{1}-\beta_{1}\right)\left(A_{2}+\gamma_{2}\right)\right) .
$$

Substituting this into (21), we have that the corresponding solutions are

$$
\begin{aligned}
& x_{1}=\frac{\left(\Delta_{2}-\sqrt{\Delta}\right)}{2 B_{2}\left(A_{1}+\beta_{1}\right)\left(A_{1}\left(A_{1}-A_{2}+\beta_{1}\right)+\beta_{1} \gamma_{2}\right)} \\
& x_{2}=\frac{\left(\Delta_{2}+\sqrt{\Delta}\right)}{2 B_{2}\left(A_{1}+\beta_{1}\right)\left(A_{1}\left(A_{1}-A_{2}+\beta_{1}\right)+\beta_{1} \gamma_{2}\right)}
\end{aligned}
$$

where

$$
\Delta_{2}:=\left(A_{1}+\beta_{1}\right)\left(-\left(A_{1}+\beta_{1}\right) \gamma_{2}^{2}-\left(\left(A_{1}+\beta_{1}\right)^{2}+B_{2} \alpha_{1}\right) \gamma_{2}+\left(-A_{1}+A_{2}-\beta_{1}\right)\left(A_{2}\left(A_{1}+\beta_{1}\right)-B_{2} \alpha_{1}\right)\right) .
$$

Claim 1 Assume $\Delta \geq 0$. Then we have:
a) If $x_{1} \geq 0$ then $y_{1}<0$.

b) If $x_{2} \geq 0$ then $y_{2}<0$.

Proof. Since $T:[0, \infty)^{2} \rightarrow[0, \infty)^{2}, T\left(x_{1}, y_{1}\right)=\left(x_{2}, y_{2}\right)$ and $T\left(x_{2}, y_{2}\right)=\left(x_{1}, y_{1}\right)$, it is obvious that if $\left(x_{i}, y_{i}\right) \in[0, \infty)^{2}$ holds then $T\left(x_{i}, y_{i}\right) \in[0, \infty)^{2}$ for $i=1$, 2. It is enough to show that the assumptions $\left(x_{1}, y_{1}\right),\left(x_{2}, y_{2}\right) \in[0, \infty)^{2}$ and $T\left(x_{1}, y_{1}\right)=\left(x_{2}, y_{2}\right) \neq\left(x_{1}\right.$, $y_{1}$ ) lead to a contradiction.

Indeed, if $A_{1}\left(A_{1}-A_{2}+\beta_{1}\right)+\beta_{1} \gamma_{2}>0$ then $\left(x_{1}, y_{1}\right) \prec_{s e}\left(x_{2}, y_{2}\right)$. Since $T$ is strongly competitive map then $\left(x_{2}, y_{2}\right)=T\left(x_{1}, y_{1}\right)<_{s e} T\left(x_{2}, y_{2}\right)=\left(x_{1}, y_{1}\right)$ which is impossible since $\left(x_{1}, y_{1}\right) \prec_{s e}\left(x_{2}, y_{2}\right)$.

If $A_{1}\left(A_{1}-A_{2}+\beta_{1}\right)+\beta_{1} \gamma_{2}<0$ then $\left(x_{2}, y_{2}\right) \prec_{s e}\left(x_{1}, y_{1}\right)$ Similarly, we have the same conclusion if $A_{1}\left(A_{1}-A_{2}+\beta_{1}\right)+\beta_{1} \gamma_{2}=0$.

\subsection{Boundedness of solutions}

Lemma 3 Assume that $y_{0}=0, x_{0} \in \mathbb{R}^{+}$. Then the following statements are true.

(i) If $A_{1}>\beta_{1}$ then $y_{n}=0 x_{n} \rightarrow \frac{\alpha_{1}}{A_{1}-\beta_{1}}, n \rightarrow \infty$.

(ii) If $A_{1}<\beta_{1}$ then $y_{n}=0, x_{n} \rightarrow \infty, n \rightarrow \infty$.

(iii) If $A_{1}=\beta_{1}$, then $x_{n}=x_{0}+\frac{\alpha_{1}}{A_{1}}$ nand $y_{n}=0, x_{n} \rightarrow \infty$.

Assume that $y_{0} \neq 0$ and $\left(x_{0}, y_{0}\right) \in \mathbb{R}_{2}^{+}$. Then the following statements are true.

(iv) $x_{n+1} \leq \frac{\alpha_{1}}{A_{1}}+\frac{\beta_{1}}{A_{1}} x_{n}$ for all $n=0,1,2, \ldots$

(v) $y_{n} \leq \gamma_{2}, n \geq N, y_{n+1} \leq C\left(\frac{\gamma_{2}}{A_{2}}\right)^{n}$ and

(a) $x_{n} \rightarrow \frac{\alpha_{1}}{A_{1}-\beta_{1}}, A_{1}>\beta_{1}$.

(b) $x_{n} \leq \frac{\alpha_{1}}{A_{1}-\beta_{1}}+\varepsilon, \varepsilon>0, A_{1}>\beta_{1}$.

(c) If $\gamma_{2}<A_{2}$ then $y_{n} \rightarrow 0, n \rightarrow \infty$

Proof. Take $y_{0}=0$ and $x_{0} \in \mathbb{R}^{+}$. Then, we have $y_{n}=0$, for all $\mathrm{n} \in \mathbb{N}$, and 


$$
x_{n+1}=\frac{\alpha_{1}}{A_{1}}+\frac{\beta_{1}}{A_{1}} x_{n} .
$$

Solution of Equation 26 is

$$
x_{n}=c\left(\frac{\beta_{1}}{A_{1}}\right)^{n}+\frac{\alpha_{1}}{A_{1}-\beta_{1}}
$$

From $y_{n+1}=\frac{\gamma_{2} y_{n}}{A_{2}+B_{2} x_{n}+y_{n}}$ it follows that $y_{n+1} \leq \frac{\gamma_{2}}{A_{2}} y_{n}, y_{n+1} \leq \gamma_{2}, n \geq 0$. The proof of Lemma 3 follows from (27).

\section{Linearized stability analysis}

The map $T$ associated to System (1) is given by

$$
T(x, y)=\left(\frac{\alpha_{1}+\beta_{1} x}{A_{1}+\gamma}, \frac{\gamma_{2} y}{A_{2}+B_{2} x+y}\right) .
$$

The Jacobian matrix of the map $T$ has the form:

$$
J_{T}=\left(\begin{array}{cc}
\frac{\beta_{1}}{A_{1}+\gamma} & -\frac{\alpha_{1}+\beta_{1} x}{\left(A_{1}+\gamma\right)^{2}} \\
-\frac{B_{2} \gamma_{2} y}{\left(A_{2}+B_{2} x+y\right)^{2}} & \frac{\gamma_{2} A_{2}+\gamma_{2} B_{2} x}{\left(A_{2}+B_{2} x+y\right)^{2}}
\end{array}\right) .
$$

The value of the Jacobian matrix of $T$ at the equilibrium point $E=(\bar{x}, \bar{y})$ is

$$
J_{T}(\bar{x}, \bar{y})=\left(\begin{array}{cc}
\frac{\beta_{1}}{A_{1}+\bar{y}} & -\frac{\bar{x}}{A_{1}+\bar{y}} \\
-\frac{B_{2} \bar{y}}{A_{2}+B_{2} \bar{x}+\bar{y}} & \frac{\gamma_{2} A_{2}+\gamma_{2} B_{2} \bar{x}}{\left(A_{2}+B_{2} \bar{x}+\bar{y}\right)^{2}}
\end{array}\right) .
$$

The determinant of (29) is given by

$$
\operatorname{det} J_{T}(\bar{x}, \bar{y})=\frac{\beta_{1}}{A_{1}+\bar{y}} \frac{\gamma_{2} A_{2}+\gamma_{2} B_{2} \bar{x}}{\left(A_{2}+B_{2} \bar{x}+\bar{y}\right)^{2}}-\frac{\bar{x}}{A_{1}+\bar{y}} \frac{B_{2} \bar{y}}{A_{2}+B_{2} \bar{x}+\bar{y}} .
$$

The trace of (29) is

$$
\operatorname{Tr} J_{T}(\bar{x}, \bar{y})=\frac{\beta_{1}}{A_{1}+\bar{y}}+\frac{\gamma_{2} A_{2}+\gamma_{2} B_{2} \bar{x}}{\left(A_{2}+B_{2} \bar{x}+\bar{y}\right)^{2}}
$$

The characteristic equation has the form

$$
\lambda^{2}-\lambda\left(\frac{\beta_{1}}{A_{1}+\bar{y}}+\frac{\gamma_{2} A_{2}+\gamma_{2} B_{2} \bar{x}}{\left(A_{2}+B_{2} \bar{x}+\bar{y}\right)}\right)+\frac{\beta_{1}\left(\gamma_{2} A_{2}+\gamma_{2} B_{2} \bar{x}\right)}{\left(A_{1}+\bar{y}\right)\left(A_{2}+B_{1} \bar{x}+\bar{y}\right)^{2}}-\frac{B_{1} \bar{x} \bar{y}}{\left(A_{1}+\bar{y}\right)\left(A_{2}+B_{2} \bar{x}+\bar{y}\right)}=0 .
$$

Theorem 9 Assume that $A_{1}>\beta_{1}$. Then there exists the equilibrium point $E_{1}$ and:

(i) $E_{1}$ is locally asymptotically stable if $\gamma_{2}-A_{2}<\frac{B_{2} \alpha_{1}}{A_{1}-\beta_{1}}$.

(ii) $E_{1}$ is a saddle point if $\gamma_{2}-A_{2}>\frac{B_{2} \alpha_{1}}{A_{1}-\beta_{1}}$. The eigenvalues are

$$
\lambda_{1}=\frac{\beta_{1}}{A_{1}}, \quad \lambda_{2}=\frac{\left(A_{1}-\beta_{1}\right) \gamma_{2}}{B_{2} \alpha_{1}+A_{2}\left(A_{2}-\beta_{1}\right)} .
$$


The corresponding eigenvectors, respectively, are

$$
v_{1}=(1,0), \quad v_{2}=\left(\frac{\alpha_{1}}{A_{1}\left(A_{1}-\beta_{1}\right)\left(\frac{\beta_{1}}{\alpha_{1}}-\frac{\left(A_{1}-\beta_{1}\right)}{A_{1} A_{2}+B_{2} \alpha_{1}-A_{2} \beta_{1}}\right)}, 1\right) .
$$

(iii) $E_{1}$ is non-hyperbolic if $\gamma_{2}-A_{2}=\frac{B_{2} \alpha_{1}}{A_{1}-\beta_{1}}$.The eigenvalues are $\lambda_{1}=\frac{\beta_{1}}{A_{1}}, \lambda_{2}=1$. The corresponding eigenvectors are $\left(-\frac{\alpha_{1}}{\left(A_{1}-\beta_{1}\right)^{2}}, 1\right)$ and $(1,0)$.

Proof. Evaluating Jacobian (29) at the equilibrium point $E_{1}\left(\alpha_{1} /\left(A_{1}-\beta_{1}\right), 0\right)$,

$$
J_{T}\left(E_{1}\right)=\left(\begin{array}{cc}
\frac{\beta_{1}}{A_{1}} & -\frac{\alpha_{1}}{A_{1}\left(A_{1}-\beta_{1}\right)} \\
0 & \frac{\left(A_{1}-\beta_{1}\right) \gamma_{2}}{A_{2}\left(A_{1}-\beta_{1}\right)+B_{2} \alpha_{1}}
\end{array}\right) .
$$

The determinant of (30) is given by

$$
\operatorname{det} J_{T}(\bar{x}, \bar{y})=\frac{\beta_{1} \gamma_{2}\left(A_{1}-\beta_{1}\right)}{A_{1}\left[A_{2}\left(A_{1}-\beta_{1}\right)+B_{2} \alpha_{1}\right]} .
$$

The trace of (30) is

$$
\operatorname{Tr} J_{T}(\bar{x}, \bar{y})=\frac{\beta_{1}}{A_{1}}+\frac{\left(A_{1}-\beta_{1}\right) \gamma_{2}}{A_{2}\left(A_{1}-\beta_{1}\right)+B_{2} \alpha_{1}} .
$$

The characteristic equation associated to System (1) at $E_{1}$ has the form

$$
\left(\frac{\beta_{1}}{A_{1}}-\lambda\right)\left(\frac{\left(A_{1}-\beta_{1}\right) \gamma_{2}}{A_{2}\left(A_{1}-\beta_{1}\right)+B_{2} \alpha_{1}}-\lambda\right)=0 .
$$

From Equation 31 we have

$$
\lambda_{1}=\frac{\beta_{1}}{A_{1}}, \quad \lambda_{2}=\frac{\left(A_{1}-\beta_{1}\right) \gamma_{2}}{A_{2}\left(A_{1}-\beta_{1}\right)+B_{2} \alpha_{1}} .
$$

(i) If $A_{1}>\beta_{1}$ and $\gamma_{2}-A_{2}<\frac{B_{2} \alpha_{1}}{A_{1}-\beta_{1}}$ then $\lambda_{1}<1$ and $\lambda_{2}<1$. Hence, $E_{1}$ is a sink.

(ii) If $A_{1}>\beta_{1}$ and $\gamma_{2}-A_{2}>\frac{B_{2} \alpha_{1}}{A_{1}-\beta_{1}}$. Then $\lambda_{1}<1$, and $\lambda_{2}<1$. Hence, $E_{1}$ is a saddle.

(iii) If $A_{1}>\beta_{1}$ and $\gamma_{2}-A_{2}=\frac{B_{2} \alpha_{1}}{A_{1}-\beta_{1}}$. Then, using Equation 31, we have that $\lambda_{1}<1$ and $\lambda_{2}<1$.

From (30) we obtain the eigenvectors that correspond to these eigenvalues.

We now perform a similar analysis for the other cases in table.

\section{Theorem 10 Assume}

$$
A_{1}>\beta_{1}, \quad A_{1}+A_{2}<\beta_{1}+\gamma_{2}, \quad \frac{\left(A_{1}-\beta_{1}\right)\left(\gamma_{2}-A_{2}\right)}{B_{2}}<\alpha_{1}<\frac{\left(A_{1}-A_{2}-\beta_{1}+\gamma_{2}\right)^{2}}{4 B_{2}} .
$$

Then $E_{1}, E_{2}, E_{3}$ exist and:

(i) Equilibrium $E_{1}$ is locally asymptotically stable. 
(ii) Equilibrium $E_{3}$ is a saddle point. The eigenvalues are

$$
\lambda_{1}=\frac{-\bar{y}_{3}\left(A_{1}+\bar{y}_{3}\right)+\gamma_{2}\left(A_{1}+\beta_{1}+\bar{y}_{3}\right)-\sqrt{\mathcal{D}}}{2 \gamma_{2}\left(A_{1}+\bar{y}_{3}\right)}
$$

and

$$
\lambda_{2}=\frac{-\bar{y}_{3}\left(A_{1}+\bar{y}_{3}\right)+\gamma_{2}\left(A_{1}+\beta_{1}+\bar{y}_{3}\right)+\sqrt{\mathcal{D}}}{2 \gamma_{2}\left(A_{1}+\bar{\gamma}_{3}\right)},
$$

and $\left|\lambda_{1}\right|<1,\left|\lambda_{2}\right|>1$, where

$$
\mathcal{D}=\bar{y}_{3}^{2}\left(A_{1}+\bar{y}_{3}\right)^{2}-2 \gamma_{2} \bar{\gamma}_{3}\left(A_{1}-\beta_{1}-2 B_{2} \bar{x}_{3}+\bar{y}_{3}\right)\left(A_{1}+\bar{\gamma}_{3}\right)+\gamma_{2}^{2}\left(A_{1}-\beta_{1}+\bar{\gamma}_{3}\right)^{2} .
$$

The corresponding eigenvectors, respectively, are

$$
\begin{gathered}
v_{1}=\left(-\bar{\gamma}_{3}\left(A_{1}+\bar{\gamma}_{3}\right)+\gamma_{2}\left(A_{1}-\beta_{1}+\bar{y}_{3}\right)+\sqrt{D}, \quad 2 B_{2} \bar{\gamma}_{3}\left(A_{1}+\bar{y}_{3}\right)\right) \\
v_{2}=\left(-\bar{\gamma}_{3}\left(A_{1}+\bar{y}_{3}\right)+\gamma_{2}\left(A_{1}-\beta_{1}+\bar{y}_{3}\right)-\sqrt{\mathcal{D}}, \quad 2 B_{2} \bar{\gamma}_{3}\left(A_{1}+\bar{y}_{3}\right)\right) .
\end{gathered}
$$

(iii) Equilibrium $E_{2}$ is locally asymptotically stable.

Proof. By Theorem 9 (i) holds.

Equilibrium $E_{3}$ is a saddle if and only if the following conditions are satisfied

$$
\left|\operatorname{Tr}_{T}(\bar{x}, \bar{y})\right|>\left|1+\operatorname{det} J_{T}(\bar{x}, \bar{y})\right| \text { and } \operatorname{Tr}^{2} J_{T}(\bar{x}, \bar{y})-4 \operatorname{det} J_{T}(\bar{x}, \bar{y})>0 .
$$

The first condition is equivalent to

$$
\frac{\beta_{1}}{A_{1}+\bar{\gamma}}+\frac{\gamma_{2} A_{2}+\gamma_{2} B_{2} \bar{x}}{\left(A_{2}+B_{2} \bar{x}+\bar{y}\right)^{2}}>\left|1+\frac{\beta_{1}}{\left(A_{1}+\bar{\gamma}\right)} \frac{\gamma_{2} A_{2}+\gamma_{2} B_{2} \bar{x}}{\left(A_{2}+B_{2} \bar{x}+\bar{\gamma}\right)^{2}}-\frac{B_{2} \bar{x} \bar{y}}{\left(A_{1}+\bar{\gamma}\right)\left(A_{2}+B_{2} \bar{x}+\bar{\gamma}\right)}\right|
$$

which is equivalent to

$$
\begin{aligned}
& \beta_{1}\left(A_{2}+B_{2} \bar{x}+\bar{y}\right)^{2}+\left(A_{1}+\bar{y}\right)\left(\gamma_{2} A_{2}+\gamma_{2} B_{2} \bar{x}\right) \\
& \quad>\left(A_{1}+\bar{y}\right)\left(A_{2}+B_{2} \bar{x}+\bar{y}\right)^{2}+\beta_{1} \gamma_{2}\left(A_{2}+B_{2} \bar{x}\right)-B_{2} \bar{x} \bar{y}\left(A_{2}+B_{2} \bar{x}+\bar{y}\right) .
\end{aligned}
$$

This is equivalent to

$$
\begin{aligned}
\left(A_{2}+B_{2} \bar{x}+\bar{y}\right)^{2}\left(\beta_{1}-A_{1}-\bar{y}\right)+\gamma_{2}\left(A_{2}+B_{2} \bar{x}\right)\left(A_{1}+\bar{y}-\beta_{1}\right) & >-B_{2} \bar{x} \bar{y}\left(A_{2}+B_{2} \bar{x}+\bar{y}\right) \\
\gamma_{2}^{2}\left(\beta_{1}-A_{1}-\bar{y}\right)+\gamma_{2}\left(A_{2}+B_{2} \bar{x}\right)\left(A_{1}+\bar{y}-\beta_{1}\right) & >-B_{2} \gamma_{2} \bar{x} \bar{y} \\
\left(A_{1}-\beta_{1}+\bar{y}\right)\left(A_{2}+B_{2} \bar{x}-\gamma_{2}\right) & >-B_{2} \bar{x} \bar{y} \\
\left(\beta_{1}-A_{1}-\bar{y}\right)\left(A_{2}+B_{2} \bar{x}-\gamma_{2}\right) & <B_{2} \bar{x} \bar{y} .
\end{aligned}
$$

We have to prove that $\left(\beta_{1}-A_{1}-\bar{y}_{3}\right)\left(A_{2}+B_{2} \bar{x}_{3}-\gamma_{2}\right)<B_{2} \bar{x}_{3} \bar{y}_{3}$. Notice that

$$
\beta_{1}-A_{1}-\bar{y}_{3}=-B_{2} \bar{x}_{2} \text { and } A_{2}+B_{2} \bar{x}_{3}-\gamma_{2}=-\bar{y}_{3} .
$$

Now,

$$
\left(\beta_{1}-A_{1}-\bar{y}_{3}\right)\left(A_{2}+B_{2} \bar{x}_{3}-\gamma_{2}\right)<B_{2} \bar{x}_{3} \bar{y}_{3}
$$

is equivalent to $B_{2} \bar{x}_{2} \bar{y}_{3}<B_{2} \bar{x}_{3} \bar{y}_{3}$. This implies $\bar{x}_{2}<\bar{x}_{3}$ which is true. Condition

$$
\operatorname{Tr}^{2} J_{T}(\bar{x}, \bar{y})-4 \operatorname{det} J_{T}(\bar{x}, \bar{y})>0
$$


is equivalent to

$$
\left(\frac{\beta_{1}}{A_{1}+\bar{y}}-\frac{\gamma_{2} A_{2}+\gamma_{2} B_{2} \bar{x}}{\left(A_{2}+B_{2} \bar{x}+\bar{y}\right)^{2}}\right)^{2}+\frac{4 B_{2} \bar{x} \bar{y}}{\left(A_{1}+\bar{y}\right)\left(A_{2}+B_{2} \bar{x}+\bar{y}\right)}>0
$$

which is clearly satisfied. Hence, $E_{3}$ is a saddle.

Now, we prove that $E_{2}$ is locally asymptotically stable. Notice that

$$
\left|\operatorname{Tr} J_{T}(\bar{x}, \bar{y})\right|<1+\operatorname{det} J_{T}(\bar{x}, \bar{y})<2
$$

implies $\bar{x}_{3}>\bar{x}_{2}$ which is true.

The second condition is equivalent to

$$
\frac{\beta_{1}}{\left(A_{1}+\bar{y}\right)} \frac{\gamma_{2} A_{2}+\gamma_{2} B_{2} \bar{x}}{\left(A_{2}+B_{2} \bar{x}+\bar{y}\right)^{2}}-\frac{B_{2} \bar{x} \bar{y}}{\left(A_{1}+\bar{y}\right)\left(A_{2}+B_{2} \bar{x}+\bar{y}\right)}<1 .
$$

This implies the following

$$
\beta_{1} \gamma_{2}\left(A_{2}+B_{2} \bar{x}\right)-B_{2} \bar{x} \bar{y}\left(A_{2}+B_{2} \bar{x}+\bar{y}\right)<\left(A_{1}+\bar{y}\right)\left(A_{2}+B_{2} \bar{x}+\bar{y}\right)^{2} .
$$

Now, using Equation 5, we obtain

$$
\begin{aligned}
\beta_{1} \gamma_{2}\left(\gamma_{2}-\bar{y}\right)-B_{2} \bar{x} \bar{y} \gamma_{2} & <\left(A_{1}+\bar{y}\right) \gamma_{2}^{2} \\
-\left(\beta_{1} \bar{y}+B_{2} \bar{x} \bar{y}\right) & <\left(A_{1}-\beta_{1}+\bar{y}\right) \gamma_{2}
\end{aligned}
$$

which is true, since the left side is always negative, while the right side is always positive.

Theorem 11 Assume

$$
A_{1}>\beta_{1}, \quad A_{1}+A_{2}<\beta_{1}+\gamma_{2}, \quad \alpha_{1}=\frac{\left(A_{1}-A_{2}-\beta_{1}+\gamma_{2}\right)^{2}}{4 B_{2}} .
$$

Then $E_{1}\left(\alpha_{1} /\left(A_{1}-\beta_{1}\right), 0\right)$ and $E_{2}=E_{3}=\left(\frac{\gamma_{2}-A_{2}+A_{1}-\beta_{1}}{2 B_{2}}, \frac{\gamma_{2}-A_{2}-A_{1}+\beta_{1}}{2}\right)$ exist and

(i) Equilibrium $E_{1}$ is locally asymptotically stable.

(ii) Equilibrium $E_{2}$ is non-hyperbolic. The eigenvalues are

$$
\lambda_{1}=1, \quad \lambda_{2}=\frac{A_{1}^{2}-A_{2}^{2}+2 A_{2} \beta_{1}-\beta_{1}^{2}+2 A_{2} \gamma_{2}+2 \beta_{1} \gamma_{2}-\gamma_{2}^{2}}{2 \gamma_{2}\left(A_{1}-A_{2}+\beta_{1}+\gamma_{2}\right)} .
$$

The corresponding eigenvectors are

$$
\left(-1 / B_{2}, 1\right), \quad\left(\frac{2 \gamma_{2}\left(A_{1}-A_{2}-\beta_{1}+\gamma_{2}\right)}{B_{2}\left(-A_{1}-A_{2}+\beta_{1}+\gamma_{2}\right)\left(A_{1}-A_{2}+\beta_{1}+\gamma_{2}\right)}, 1\right) .
$$

Proof. By Theorem 9, $E_{1}$ is locally asymptotically stable.

Now, we prove that $E_{2}$ is non-hyperbolic.

Evaluating Jacobian (29) at the equilibrium point $E_{2}=\left(\frac{\gamma_{2}-A_{2}+A_{1}-\beta_{1}}{2 B_{2}}, \frac{\gamma_{2}-A_{2}-A_{1}+\beta_{1}}{2}\right)$,

$$
J_{T}\left(E_{2}\right)=\left(\begin{array}{cc}
\frac{\beta_{1}}{A_{1}+\bar{y}} & -\frac{\bar{x}}{A_{1}+\bar{y}} \\
-\frac{B_{2} \bar{y}}{\gamma_{2}} & \frac{A_{2}+B_{2} \bar{x}}{\gamma_{2}}
\end{array}\right)=\left(\begin{array}{cc}
\frac{2 \beta_{1}}{A_{1}+\gamma_{2}-A_{2}+\beta_{1}} & \frac{-\gamma_{2}+A_{2}-A_{1}+\beta_{1}}{B_{2}\left(A_{1}+\gamma_{2}-A_{2}+\beta_{1}\right)} \\
\frac{-B_{2}\left(\gamma_{2}-A_{2}-A_{1}+\beta_{1}\right)}{2 \gamma_{2}} & \frac{A_{2}+\gamma_{2}+A-1-\beta_{1}}{2 \gamma_{2}}
\end{array}\right) .
$$


The eigenvalues of (32) are

$$
\lambda_{1}=1, \quad \text { and } \quad \lambda_{2}=\frac{A_{1}^{2}-A_{2}^{2}+2 A_{2} \beta_{1}-\beta_{1}^{2}+2 A_{2} \gamma_{2}+2 \beta_{1} \gamma_{2}-\gamma_{2}^{2}}{2 \gamma_{2}\left(A_{1}-A_{1}+\beta_{1}+\gamma_{2}\right)} .
$$

Notice that $\left|\lambda_{2}\right|<1$. Hence, $E_{2}$ is non-hyperbolic.

Theorem 12 Assume

$$
\begin{aligned}
& A_{1}>\beta_{1}, \quad A_{2}<\gamma_{2}<A_{1}+A_{2}-\beta_{1}, \quad \frac{\left(A_{1}-\beta_{1}\right)\left(\gamma_{2}-A_{2}\right)}{B_{2}}<\alpha_{1} \leq \frac{\left(A_{1}-A_{2}-\beta_{1}+\gamma_{2}\right)^{2}}{4 B_{2}} \\
& A_{1}>\beta_{1}, \quad A_{2}>\gamma_{2}, \quad \alpha_{1} \leq \frac{\left(A_{1}-A_{2}-\beta_{1}+\gamma_{2}\right)^{2}}{4 B_{2}}, \quad A_{1}+\gamma_{2} \neq A_{2}+\beta_{1} \\
& A_{1}>\beta_{1}, \quad A_{2}=\gamma_{2}, \quad \alpha_{1} \leq \frac{\left(A_{1}-A_{2}-\beta_{1}+\gamma_{2}\right)^{2}}{4 B_{2}} \\
& A_{1}>\beta_{1}, \quad \alpha_{1}>\frac{\left(A_{1}-A_{2}-\beta_{1}+\gamma_{2}\right)^{2}}{4 B_{2}}
\end{aligned}
$$

Then there exists a unique equilibrium $E_{1}\left(\alpha_{1} /\left(A_{1}-\beta\right), 0\right)$ which is locally asymptotically stable.

Proof. Observe that the assumption of Theorem 12 implies that the $y$ coordinates of the equilibrium $E_{2}$ and $E_{3}$ are less then zero. By Theorem $9 E_{1}$ is locally asymptotically stable.

Theorem 13 Assume

$$
A_{1}>\beta_{1}, \quad A_{2}<\gamma_{2}, \quad \alpha_{1}<\frac{\left(A_{1}-\beta_{1}\right)\left(\gamma_{2}-A_{2}\right)}{B_{2}} .
$$

Then then there exist two equilibrium points $E_{1}$ and $E_{2} . E_{1}$ is a saddle point. The eigenvalues are

$$
\lambda_{1}=\frac{\beta_{1}}{A_{1}}, \quad \lambda_{2}=\frac{\left(A_{1}-\beta_{1}\right) \gamma_{2}}{B_{2} \alpha_{1}+A_{2}\left(A_{2}-\beta_{1}\right)} .
$$

The corresponding eigenvectors, respectively, are

$$
v_{1}=(1,0), \quad v_{2}=\left(\frac{\alpha_{1}}{A_{1}\left(A_{1}-\beta_{1}\right)\left(\frac{\beta_{1}}{\alpha_{1}}-\frac{\left(A_{1}-\beta_{1}\right)}{A_{1} A_{2}+B_{2} \alpha_{1}-A_{2} \beta_{1}}\right)}, 1\right) \text {. }
$$

The equilibrium $E_{2}$ is locally asymptotically stable.

Proof. By Theorem 9 (ii), $E_{1}$ is a saddle point.

Now, we check the sign of coordinates of the equilibrium point $E_{2}$. We have that $\bar{x}_{2}>0$, since all parameters are positive. Consider $\bar{y}_{2}$. Since

$$
\frac{\left(A_{1}-A_{2}-\beta_{1}+\gamma_{2}\right)^{2}}{4 B_{2}}-\frac{\left(A_{1}-\beta_{1}\right)\left(\gamma_{2}-A_{2}\right)}{B_{2}}=\frac{\left(A_{1}+A_{2}-\beta_{1}-\gamma_{2}\right)^{2}}{4 B_{2}}>0,
$$

we have that $\left(\gamma_{2}-A_{2}+A_{1}-\beta_{1}\right)^{2}-4 \alpha_{1} B_{2}>0$.

$$
\bar{y}_{1}>0 \Leftrightarrow \gamma_{2}-A_{2}+\beta_{1}-A_{1}+\sqrt{\left(\gamma_{2}-A_{2}+A_{1}-\beta_{1}\right)^{2}-4 \alpha_{1} B_{2}}>0 .
$$

This implies

$$
\sqrt{\left(\gamma_{2}-A_{2}+A_{1}-\beta_{1}\right)^{2}-4 \alpha_{1} B_{2}}>\left(A_{1}-\beta_{1}\right)-\left(\gamma_{2}-A_{2}\right) .
$$


From Equation 33, we see that inequality is always true if $A_{1}-\beta_{1}<\gamma_{2}-A_{2}$. If $A_{1}-\beta_{1}$ $>\gamma_{2}-A_{2}$, then

$$
\begin{aligned}
\left(\gamma_{2}-A_{2}\right)^{2}+2\left(\gamma_{2}-A_{2}\right)\left(A_{1}-\beta_{1}\right)+ & \left(A_{1}-\beta_{1}\right)^{2}-4 \alpha_{1} B_{1}>\left(A_{1}-\beta_{1}\right)^{2}-2\left(A_{1}-\beta_{1}\right)\left(\gamma_{2}-A_{2}\right) \\
\left(\gamma_{2}-A_{2}\right)\left(A_{1}-\beta_{1}\right) & >\alpha_{1} B_{2}
\end{aligned}
$$

which is true, since $A_{1}-\beta_{1}>\frac{B_{2} \alpha_{1}}{\gamma_{2}-A_{2}}$. So, in both cases $\bar{x}_{2}>0$ and $\bar{y}_{2}>0$.

Notice, that $\bar{x}_{3}>0$. Now, we check the sign of $\bar{y}_{3}$. Assume that $\bar{y}_{3}>0$. Then, we have

$$
\begin{aligned}
\bar{y}_{2}>0 & \Leftrightarrow\left(\gamma_{2}-A_{2}\right)-\left(A_{1}-\beta_{1}\right)>\sqrt{\left(\gamma_{2}-A_{2}+A_{1}-\beta_{1}\right)^{2}-4 \alpha_{1} B_{2} .} \\
& \Leftrightarrow\left(\gamma_{2}-A_{2}\right)\left(A-1-\beta_{1}\right)<\alpha_{1} B_{2} .
\end{aligned}
$$

This is a contradiction with the assumption of theorem and so $E_{3}$ is not in considered domain.

By Theorem $10, E_{2}$ is a locally asymptotically stable.

\section{Theorem 14 Assume}

$$
A_{1}>\beta_{1}, \quad A_{1}+A_{2}<\beta_{1}+\gamma_{2}, \quad \alpha_{1}=\frac{\left(A_{1}-\beta_{1}\right)\left(\gamma_{2}-A_{2}\right)}{B_{2}} .
$$

Then there exist two equilibrium points $E_{1} \equiv E_{3}=\left(\frac{\alpha_{1}}{A_{1}-\beta_{1}}, 0\right)$ and $E_{2}=\left(\frac{\alpha_{1}}{\gamma_{2}-A_{2}}, \frac{\gamma_{2}-A_{2}-A_{1}+\beta_{1}}{2}\right)$, and $E_{1} \equiv E_{3}$ is non-hyperbolic. The eigenvalues are $\lambda_{1}=\frac{\beta_{1}}{A_{1}}$, $\lambda_{2}=1$. The corresponding eigenvectors are $\left(-\frac{\alpha_{1}}{\left(A_{1}-\beta_{1}\right)^{2}}, 1\right)$ and (1.0) The equilibrium point $E_{2}$ is locally asymptotically stable.

Proof. By Theorem 10, $E_{2}$ is locally asymptotically stable. By Theorem 9 (iii), $E_{1}$ is non-hyperbolic.

Now, we consider the special case of System (1) when $A_{1}=\beta_{1}$.

In this case, System (1) becomes

$$
\left\{\begin{array}{l}
x_{n+1}=\frac{\alpha_{1}+A_{1} x_{n}}{A_{1}+y_{n}} \\
y_{n+1}=\frac{\gamma_{2} y_{n}}{A_{2}+B_{2} x_{n}+y_{n}}
\end{array}, \quad n=0,1,2, \ldots\right.
$$

The map $T$ associated to System (34) is given by

$$
T(x, y)=\left(\frac{\alpha_{1}+A_{1} x}{A_{1}+y}, \frac{\gamma_{2} y}{A_{2}+B_{2} x+y}\right) .
$$

The Jacobian matrix of the map $T$ has the form:

$$
J_{T}=\left(\begin{array}{cc}
\frac{A_{1}}{A_{1}+\gamma} & -\frac{\alpha_{1}+A_{1} x}{\left(A_{1}+\gamma\right)^{2}} \\
-\frac{\beta_{2} \gamma_{2} y}{\left(A_{2}+B_{2} x+y\right)^{2}} & \frac{\gamma_{2} A_{2}+\gamma_{2} B_{2} x}{\left(A_{2}+B_{2} x+y\right)^{2}}
\end{array}\right) .
$$

The value of the Jacobian matrix of $T$ at the equilibrium point $E=(\bar{x}, \bar{y})$ is

$$
J_{T}(\bar{x}, \bar{y})=\left(\begin{array}{cc}
\frac{A_{1}}{A_{1}+\bar{y}} & -\frac{\bar{x}}{A_{1}+\bar{y}} \\
-\frac{B_{2} \bar{y}}{A_{2}+B_{2} \bar{x}+\bar{y}} & \frac{\gamma_{2} A_{2}+\gamma_{2} B_{2} \bar{x}}{\left(A_{2}+B_{2} \bar{x}+\bar{y}\right)^{2}}
\end{array}\right)=\left(\begin{array}{cc}
\frac{A_{1}}{A_{1}+\bar{y}} & -\frac{\bar{x}}{A_{1}+\bar{y}} \\
-\frac{B_{2} \bar{y}}{\gamma_{2}} & \frac{A_{2}+B_{2} \bar{x}}{\gamma_{2}}
\end{array}\right) .
$$


The characteristic equation of $T$ at $(\bar{x}, \bar{y})$ has the form

$$
\lambda^{2}-\lambda\left(\frac{A_{1}}{A_{1}+\bar{y}}+\frac{A_{2}+B_{2} \bar{x}}{\gamma_{2}}\right)+\frac{A_{1}}{A_{1}+\bar{y}} \frac{A_{2}+B_{2} \bar{x}}{\gamma_{2}}-\frac{B_{2} \bar{x} \bar{y}}{\left(A_{1}+\bar{y}\right) \gamma_{2}}=0 .
$$

Equilibrium points satisfy the following System

$$
\begin{aligned}
& \bar{x}=\frac{\alpha_{1}+A_{1} \bar{x}}{A_{1}+\bar{y}} \\
& \bar{y}=\frac{\gamma_{2} \bar{y}}{A_{2}+B_{2} \bar{x}+\bar{y}} \quad n=0,1, \ldots
\end{aligned}
$$

Notice, if $\bar{y}=0$, then using the first equation of System (37 we obtain $\alpha_{1}=0$ which is impossible. If $\bar{y} \neq 0$ then, using System (37), we obtain

$$
\begin{aligned}
& \bar{y}=\gamma_{2}-A_{2}-B_{2} \bar{x} \\
& 0=B_{2} \bar{x}^{2}-\bar{x}\left(\gamma_{2}-A_{2}\right)+\alpha_{1} .
\end{aligned}
$$

and the equilibrium points are:

$$
\begin{aligned}
& E_{3}=\left(\frac{\gamma_{2}-A_{2}+\sqrt{\left(\gamma_{2}-A_{2}\right)^{2}-4 B_{2} \alpha_{1}}}{2 B_{2}}, \frac{\gamma_{2}-A_{2}-\sqrt{\left(\gamma_{2}-A_{2}\right)^{2}-4 B_{2} \alpha_{1}}}{2}\right), \\
& E_{2}=\left(\frac{\gamma_{2}-A_{2}-\sqrt{\left(\gamma_{2}-A_{2}\right)^{2}-4 B_{2} \alpha_{1}}}{2 B_{2}}, \frac{\gamma_{2}-A_{2}+\sqrt{\left(\gamma_{2}-A_{2}\right)^{2}-4 B_{2} \alpha_{1}}}{2}\right) .
\end{aligned}
$$

We prove the following.

Theorem 15 Assume

$$
A_{1}=\beta_{1} \text {. }
$$

Then the following statements hold.

(i) If $\gamma_{2}>A_{2},\left(\gamma_{2}-A_{2}\right)^{2}-4 B_{2} \alpha_{1}>0$ then System (34) has two positive equilibrium points

$$
E_{3}=\left(\frac{\gamma_{2}-A_{2}+\sqrt{\left(\gamma_{2}-A_{2}\right)^{2}-4 B_{2} \alpha_{1}}}{2 B_{2}}, \frac{\gamma_{2}-A_{2}-\sqrt{\left(\gamma_{2}-A_{2}\right)^{2}-4 B_{2} \alpha_{1}}}{2}\right)
$$

and

$$
E_{2}=\left(\frac{\gamma_{2}-A_{2}-\sqrt{\left(\gamma_{2}-A_{2}\right)^{2}-4 B_{2} \alpha_{1}}}{2 B_{2}}, \frac{\gamma_{2}-A_{2}+\sqrt{\left(\gamma_{2}-A_{2}\right)^{2}-4 B_{2} \alpha_{1}}}{2}\right) .
$$

$E_{3}$ is a saddle point. The eigenvalues are

$$
\begin{array}{ll}
\lambda_{1}=\frac{-\bar{y}_{3}\left(A_{1}+\bar{y}_{3}\right)+\gamma_{2}\left(2 A_{1}+\bar{y}_{3}\right)-\sqrt{F}}{2 \gamma_{2}\left(A_{1}+\bar{y}_{3}\right)}, & \left|\lambda_{1}\right|<1 \\
\lambda_{2}=\frac{-\bar{y}_{3}\left(A_{1}+\bar{y}_{3}\right)+\gamma_{2}\left(2 A_{1}+\bar{y}_{3}\right)+\sqrt{F}}{2 \gamma_{2}\left(A_{1}+\bar{y}_{3}\right)}, & \lambda_{2}>1,
\end{array}
$$


where

$$
F=\bar{y}_{3}^{2}\left(A_{1}+\bar{y}_{3}\right)^{2}-2 \gamma_{2} \bar{\gamma}_{3}\left(\bar{y}_{3}-2 B_{2} \bar{x}_{3}\right)\left(A_{1}+\bar{y}_{3}\right)+\gamma_{2}^{2} \bar{y}_{3}^{2} .
$$

The corresponding eigenvectors are

$$
\begin{aligned}
& v_{1}=\left(-\bar{y}_{3}\left(A_{1}+\bar{y}_{3}\right)+\gamma_{2} \bar{y}_{3}+\sqrt{F}, 2 B_{2} \bar{y}_{3}\left(A_{1}+\bar{y}_{3}\right)\right), \\
& v_{2}=\left(-\bar{y}_{3}\left(A_{1}+\bar{y}_{3}\right)+\gamma_{2} \bar{y}_{3}-\sqrt{F}, 2 B_{2} \bar{y}_{3}\left(A_{1}+\bar{y}_{3}\right)\right) .
\end{aligned}
$$

The equilibrium $E_{2}$ is locally asymptotically stable.

(ii) If $\gamma_{2}>A_{2},\left(\gamma_{2}-A_{2}\right)^{2}-4 B_{2} \alpha_{1}>0$ then System (34) has a unique equilibrium point $E=\left(\frac{\gamma_{2}-A_{2}}{2 B_{2}}, \frac{\gamma_{2}-A_{2}}{2}\right)$ which is non-hyperbolic. The eigenvalues are $\lambda_{1}=1$ and $\lambda_{2}=\frac{2 A_{1} A_{2}-A_{2}^{2}+2 A_{1} \gamma_{2}+2 A_{2} \gamma_{2}-\gamma_{2}^{2}}{2 \gamma_{2}\left(2 A_{1}-A_{2}+\gamma_{2}\right)}$. The corresponding eigenvectors are: $\left(-1 / B_{2}, 1\right)$ and $\left(\frac{2 \gamma_{2}}{B_{2}\left(2 A_{1}-A_{2}+\gamma_{2}\right)}, 1\right)$.

(iii) If $\gamma_{2}<A_{2}$ and $\left(\gamma_{2}-A_{2}\right)^{2}-4 B_{2} \alpha_{1} \geq 0$ or $\left(\gamma_{2}-A_{2}\right)^{2}-4 B_{2} \alpha_{1}>0$ then System (34) has no equilibrium points.

Proof. (i) First, notice that under these assumptions, $E_{3}$ and $E_{2}$ are positive. Now, we prove that $E_{3}$ is a saddle point.

The equilibrium point $E_{3}$ is a saddle if and only if the following conditions are satisfied $\left|\operatorname{Tr} J_{T}(\bar{x}, \bar{y})\right|>\left|1+\operatorname{det} J_{T}(\bar{x}, \bar{y})\right|$ and $\operatorname{Tr}^{2} J_{T}(\bar{x}, \bar{y})-4 \operatorname{det} J_{T}(\bar{x}, \bar{y})>0$.

The first condition is equivalent to

$$
\frac{A_{1}}{A_{1}+\bar{y}}+\frac{A_{2}+B_{2} \bar{x}}{\gamma_{2}}>\left|1+\frac{A_{1}\left(A_{2}+B_{2} \bar{x}\right)}{\gamma_{2}\left(A_{1}+\bar{y}\right)}-\frac{B_{2} \bar{x} \bar{y}}{\gamma_{2}\left(A_{1}+\bar{y}\right)}\right|,
$$

which is equivalent to

$$
A_{1} \gamma_{2}+\left(A_{1}+\bar{y}\right)\left(A_{2}+B_{2} \bar{x}\right)>\gamma_{2}\left(A_{1}+\bar{y}\right)+A_{1}\left(A_{1}+B_{2} \bar{x}\right)-B_{2} \bar{x} \bar{y}
$$

and this is equivalent to

$$
\gamma_{2}-A_{2}<2 B_{2} \bar{x} \text {. }
$$

In the case of equilibrium $E_{3}$, this condition becomes

$$
\gamma_{2}-A_{2}<2 B_{2} \bar{x}_{3} \Leftrightarrow \gamma_{2}-A_{2}<\gamma_{2}-A_{2}+\sqrt{\left(\gamma_{2}-A_{2}\right)^{2}-4 B_{2} \alpha_{1}} \Leftrightarrow \sqrt{\left(\gamma_{2}-A_{2}\right)^{2}-4 B_{2} \alpha_{1}}>0,
$$

which is true.

The second condition becomes

$$
\left(\frac{A_{1}}{A_{1}+\bar{\gamma}}+\frac{A_{2}+B_{2} \bar{x}}{\gamma_{2}}\right)^{2}-4 \frac{A_{1}\left(A_{2}+B-2 \bar{x}\right)}{\gamma_{2}\left(A_{1}+\bar{y}\right)}+4 \frac{B_{2} \bar{x} \bar{y}}{\gamma_{2}\left(A_{1}+\bar{\gamma}\right)}=\left(\frac{A_{1}}{A_{1}+\bar{y}}-\frac{A_{2}+B_{2} \bar{x}}{\gamma_{2}}\right)^{2}+4 \frac{B_{2} \bar{x} \bar{y}}{\gamma_{2}\left(A_{1}+\bar{y}\right)}
$$

which is greater then zero. Hence, $E_{3}$ is a saddle.

Now, we prove that $E_{2}$ is locally asymptotically stable. The equilibrium point $E_{2}$ is locally asymptotically stable if the following is satisfied

$$
\left|\operatorname{Tr} J_{T}(\bar{x}, \bar{y})\right|<1+\operatorname{det} J_{T}(\bar{x}, \bar{y})<2 .
$$


The first condition is equivalent to

$$
\frac{A_{1}}{A_{1}+\bar{y}}+\frac{A_{2}+B_{2} \bar{x}}{\gamma_{2}}<1+\frac{A_{1}\left(A_{2}+B_{2} \bar{x}\right)}{\gamma_{2}\left(A_{1}+\bar{y}\right)}-\frac{B_{2} \bar{x} \bar{y}}{\gamma_{2}\left(A_{1}+\bar{y}\right)} \text {. }
$$

This implies

$$
A_{1} \gamma_{2}+\left(A_{1}+\bar{y}\right)\left(A_{2}+B_{2} \bar{x}\right)<\gamma_{2}\left(A_{1}+\bar{y}\right)+A_{1}\left(A_{2}+B_{2} \bar{x}\right)-B 2 \bar{x} \bar{y}
$$

which is equivalent to $\gamma_{2}-A_{2}>2 B_{2} \bar{x}$. In the case of the equilibrium point $E_{2}$, we have

$$
\gamma_{2}-A_{2}>\gamma_{2}-A_{2}-\sqrt{\left(\gamma_{2}-A_{2}\right)^{2}-4 B_{2} \alpha_{1}} \Leftrightarrow-\sqrt{\left(\gamma_{2}-A_{2}\right)^{2}-4 B_{2} \alpha_{1}}<0
$$

which is true.

The second condition is equivalent to

$$
\frac{A_{1}\left(A_{2}+B_{2} \bar{x}\right)}{\gamma_{2}\left(A_{1}+\bar{y}\right)}-\frac{B_{2} \bar{x} \bar{y}}{\gamma_{2}\left(A_{1}+\bar{y}\right)}<1 \text {. }
$$

This implies

$$
A_{1}\left(A_{2}+B_{2} \bar{x}\right)-B_{2} \bar{x} \bar{y}<\gamma_{2}\left(A_{1}+\bar{y}\right) \Leftrightarrow A_{1}\left(A_{2}-\gamma_{2}+B_{2} \bar{x}\right)<\bar{y}\left(\gamma_{2}+B_{2} \bar{x}\right) .
$$

Notice that

$$
A_{2}-\gamma_{2}+B_{2} \bar{x}_{2}=\frac{A_{2}-\gamma_{2}-\sqrt{\left(\gamma_{2}-A_{2}\right)^{2}-4 B_{2} \alpha_{1}}}{2}=-\frac{\gamma_{2}-A_{2}+\sqrt{\left(\gamma_{2}-A_{2}\right)^{2}-4 B_{2} \alpha_{1}}}{2}=-\bar{\gamma}_{2} .
$$

Now, condition $\quad A_{1}\left(A_{2}-\gamma_{2}+B_{2} \bar{x}\right)<\bar{y}\left(\gamma_{2}+B-2 \bar{x}\right) \quad$ becomes $-A_{1} \bar{y}_{2}<\bar{y}_{2}\left(\gamma_{2}+B_{2} \bar{x}_{2}\right) \Leftrightarrow-A_{1}<\gamma_{2}+B_{2} \bar{x}_{2}$ which is true. Hence, $E_{2}$ is locally asymptotically stable.

(ii) The characteristic equation associated to System (37) at $E$ has the form

$$
\lambda^{2}-\lambda\left(\frac{2 A_{1}}{2 A_{1}+\gamma_{2}-A_{2}}+\frac{A_{2}+\gamma_{2}}{2 \gamma_{2}}\right)+\frac{A_{1}}{\gamma_{2}}-\frac{\left(\gamma_{2}-A_{2}\right)^{2}}{2 \gamma_{2}\left(2 A_{1}+\gamma_{2}-A_{2}\right)}=0 .
$$

Solutions of Equation (38) are $\lambda_{1}=1$ and $\lambda_{2}=\frac{2 A_{1} A_{2}-A_{2}^{2}+2 A_{1} \gamma_{2}+2 A_{2} \gamma_{2}-\gamma_{2}^{2}}{2 \gamma_{2}\left(2 A_{1}-A_{2}+\gamma_{2}\right)}$.

The corresponding eigenvectors are $\left(-1 / B_{2}, 1\right)$ and $\left(\frac{2 \gamma_{2}}{B_{2}\left(2 A_{1}-A_{2}+\gamma_{2}\right)}, 1\right)$.

If $\gamma_{2}<A_{2}$ and $\left(\gamma_{2}-A_{2}\right)^{2}-4 B_{2} \alpha_{1} \geq 0$ then $\bar{x}_{2}<0$ and $\bar{x}_{3}<0$.

\section{Theorem 16 Assume}

$$
A_{1}<\beta_{1}, \gamma_{2}>A_{2}, \gamma_{2}-A_{2}>\beta_{1}-A_{1} \quad \text { and } \quad\left(\gamma_{2}-A_{2}+A_{1}-\beta_{1}\right)^{2}-4 B_{2} \alpha_{1}>0 \text {. }
$$

Then there exist two positive equilibrium points

$$
E_{2}=\left(\frac{\gamma_{2}-A_{2}+A_{1}-\beta_{1}-\sqrt{\left(\gamma_{2}-A_{2}+A_{1}-\beta_{1}\right)^{2}-4 B_{2} \alpha_{1}}}{2 B_{2}}, \frac{\gamma_{2}-A_{2}-A_{1}+\beta_{1}+\sqrt{\left(\gamma_{2}-A_{2}+A_{1}-\beta_{1}\right)^{2}-4 B_{2} \alpha_{1}}}{2}\right)
$$

and

$$
E_{3}=\left(\frac{\gamma_{2}-A_{2}+A_{1}-\beta_{1}+\sqrt{\left(\gamma_{2}-A_{2}+A_{1}-\beta_{1}\right)^{2}-4 B_{2} \alpha_{1}}}{2 B_{2}}, \frac{\gamma_{2}-A_{2}+\beta_{1}-A_{1}-\sqrt{\left(\gamma_{2}-A_{2}+A_{1}-\beta_{1}\right)^{2}-4 B_{2} \alpha_{1}}}{2}\right)
$$


$E_{2}$ is locally asymptotically stable and $E_{3}$ is a saddle. The eigenvalues of characteristic equation at $E_{3}$ are

$$
\lambda_{1}=\frac{-\bar{y}_{3}\left(A_{1}+\bar{y}_{3}\right)+\gamma_{2}\left(A_{1}+\beta_{1}+\bar{y}_{3}\right) \mp \sqrt{\mathcal{D}}}{2 \gamma_{2}\left(A_{1}+\bar{y}_{3}\right)},
$$

where

$$
\mathcal{D}=\bar{y}_{3}^{2}\left(A_{1}+\bar{y}_{3}\right)^{2}-2 \gamma_{2} \bar{y}_{3}\left(A_{1}-\beta_{1}-2 B_{2} \bar{x}_{3}+\bar{y}_{3}\right)\left(A_{1}+\bar{y}_{3}\right)+\gamma_{2}^{2}\left(A_{1}-\beta_{1}+\bar{y}_{3}\right)^{2} .
$$

The corresponding eigenvectors are

$$
v_{1,2}=\left(-\bar{\gamma}_{3}\left(A_{1}+\bar{\gamma}_{3}\right)+\gamma_{2}\left(A_{1}-\beta_{1}+\bar{\gamma}_{3}\right) \pm \sqrt{D}, \quad 2 B_{2} \bar{\gamma}_{3}\left(A_{1}+\bar{y}_{3}\right)\right) .
$$

Proof. Now, we prove that $E_{2}$ is a sink. We check the condition $\left|\operatorname{Tr}_{T}(\bar{x}, \bar{y})\right|<1+\operatorname{det} J_{T}(\bar{x}, \bar{y})<2$. The first condition is equivalent to

$$
\frac{\beta_{1}}{A_{1}+\bar{y}}+\frac{A_{2}+B_{2} \bar{x}}{\gamma_{2}}<1+\frac{\beta_{1}\left(A_{2}+B_{2} \bar{x}\right)}{\gamma_{2}\left(A_{1}+\bar{y}\right)}-\frac{B_{2} \bar{x} \bar{y}}{\gamma_{2}\left(A_{1}+\bar{y}\right)} .
$$

This implies

$$
\begin{aligned}
& \beta_{1} \gamma_{2}+\left(A_{1}+\bar{y}\right)\left(A_{2}+B_{2} \bar{x}\right)<\gamma_{2}\left(A_{1}+\bar{y}\right)+\beta_{1}\left(A_{2}+B_{2} \bar{x}\right)-B_{2} \bar{x} \bar{y} \\
& \gamma_{2}\left(\beta_{1}-A_{1}-\bar{y}\right)+\left(A_{2}+B_{2} \bar{x}\right)\left(A_{1}+\bar{y}-\beta_{1}\right)<-B_{2} \bar{x} \bar{y} \\
& \left(A_{1}-\beta_{1}+\bar{y}\right)\left(A_{2}+B_{2} \bar{x}-\gamma_{2}\right)<-B_{2} \bar{x} \bar{y} \\
& \bar{y}\left(A_{1}-\beta_{1}+\bar{y}\right)>B_{2} \bar{x} \bar{y} \\
& \left(A_{1}-\beta_{1}+\bar{y}\right)>B_{2} \bar{x} .
\end{aligned}
$$

So, we have to prove

$$
\left(A_{1}-\beta_{1}+\bar{y}_{2}\right)>B_{2} \bar{x}_{2} .
$$

Note that

$$
\begin{aligned}
A_{1}-\beta_{1}+\bar{\gamma}_{2} & =A_{1}-\beta_{1}+\frac{\gamma_{2}-A_{2}+\beta_{1}-A_{1}+\sqrt{\left(\gamma_{2}-A_{2}+A_{1}-\beta_{1}\right)^{2}-4 B_{2} \alpha_{1}}}{2} \\
& =\frac{A_{1}-\beta_{1}+\gamma_{2}-A_{2}+\sqrt{\left(\gamma_{2}-A_{2}+A_{1}-\beta_{1}\right)^{2}-4 B_{2} \alpha_{1}}}{2 B_{2}} B_{2} \\
& =B_{2} \bar{x}_{3} .
\end{aligned}
$$

Now, (39) becomes $B_{2} \bar{x}_{3}>B_{2} \bar{x}_{2} \Rightarrow \bar{x}_{3}>\bar{x}_{2}$ which is true.

The second condition is equivalent to

$$
\frac{\beta_{1}\left(A_{2}+B_{2} \bar{x}\right)}{\gamma_{2}\left(A_{1}+\bar{y}\right)}-\frac{B_{2} \bar{x} \bar{y}}{\gamma_{2}\left(A_{1}+\bar{y}\right)}<1 \text {. }
$$

This implies $\beta_{1}\left(\gamma_{2}-\bar{y}\right)-B_{2} \bar{x} \bar{y}<\gamma_{2}\left(A_{1}+\bar{\gamma}\right)$. Using equations of equilibrium points, we obtain $\bar{y}_{2}\left(\beta_{1}+B_{2} \bar{x}_{2}\right)>\gamma_{2}\left(\beta_{1}-A_{1}-\bar{y}_{2}\right)$ and 


$$
\begin{aligned}
\beta_{1}+B_{2} \bar{x}_{2} & =\beta_{1}+\frac{\gamma_{2}-A_{2}+A_{1}-\beta_{1}-\sqrt{\left(\gamma_{2}-A_{2}+A_{1}-\beta_{1}\right)^{2}-4 B_{2} \alpha_{1}}}{2} \\
& =\frac{\gamma_{2}-A_{2}+A_{1}+\beta_{1}-\sqrt{\left(\gamma_{2}-A_{2}+A_{1}-\beta_{1}\right)^{2}-4 B_{2} \alpha_{1}}}{2}>0 .
\end{aligned}
$$

On the other hand, we have

$$
\begin{aligned}
\left(\beta_{1}-A_{1}-\bar{y}_{2}=\right. & \beta_{1}-A_{1}-\frac{\gamma_{2}-A_{2}+\beta_{1}-A_{1}+\sqrt{\left(\gamma_{2}-A_{2}+A_{1}-\beta_{1}\right)^{2}-4 B_{2} \alpha_{1}}}{2} \\
& =\frac{\beta_{1}-A_{1}+A_{2}-\gamma_{2}-\sqrt{\left(\gamma_{2}-A_{2}+A_{1}-\beta_{1}\right)^{2}-4 B_{2} \alpha_{1}}}{2}<0
\end{aligned}
$$

since $\gamma_{2}-A_{2}>\beta_{1}-A_{1}$. Hence, $E_{2}$ is locally asymptotically stable.

Now, we prove that $E_{3}$ is a saddle.

The equilibrium point $E_{3}$ is a saddle if and only if the following conditions are satisfied

$$
\left|\operatorname{Tr} J_{T}(\bar{x}, \bar{y})\right|>\left|1+\operatorname{det} J_{T}(\bar{x}, \bar{y})\right| \quad \text { and } \quad \operatorname{Tr}^{2} J_{T}(\bar{x}, \bar{y})-4 \operatorname{det} J_{T}(\bar{x}, \bar{y})>0 .
$$

Note that the first condition is equivalent to $B_{2} \bar{x}_{3}>B_{2} \bar{x}_{2} \Rightarrow \bar{x}_{3}>\bar{x}_{2}$ which is true.

The second condition becomes

$$
\left(\frac{\beta_{1}}{A_{1}+\bar{y}}+\frac{A_{2}+B_{2} \bar{x}}{\gamma_{2}}\right)^{2}-4 \frac{\beta_{1}\left(A_{2}+B_{2} \bar{x}\right)}{\left(A_{1}+\bar{y}\right) \gamma_{2}}+4 \frac{B_{2} \bar{x} \bar{y}}{\gamma_{2}\left(A_{1}+\bar{y}\right)}=\left(\frac{\beta_{1}}{A_{1}+\bar{y}}-\frac{A_{2}+B_{2} \bar{x}}{\gamma_{2}}\right)^{2}+4 \frac{B_{2} \bar{x} \bar{y}}{\gamma_{2}\left(A_{1}+\bar{y}\right)}>0 .
$$

Hence, $E_{3}$ is a saddle.

\section{Theorem 17 Assume}

$$
A_{1}<\beta_{1}, \quad \gamma_{2}>A_{2}, \quad \gamma_{2}-A_{2}>\beta_{1}-A_{1} \quad \text { and } \quad\left(\gamma_{2}+A_{1}-A_{2}-\beta_{1}\right)^{2}-4 \alpha_{1} B_{2}=0 .
$$

Then there exists a unique equilibrium point $E_{2} \equiv E_{3}=E=\left(\frac{\gamma_{2}-A_{2}+A_{1}-\beta_{1}}{2 B_{2}}, \frac{\gamma_{2}-A_{2}+\beta_{1}-A_{1}}{2}\right)$ which is non-hyperbolic. The eigenvalues are:

$$
\lambda_{1}=1 \quad \text { and } \quad \lambda_{2}=\frac{A_{1}^{2}-A_{2}^{2}+2 A_{2} \beta_{1}-\beta_{1}^{2}+2 A_{2} \gamma_{2}+2 \beta_{1} \gamma_{2}-\gamma_{2}^{2}}{2 \gamma_{2}\left(A_{1}-A_{2}+\beta_{1}+\gamma_{2}\right)} .
$$

The corresponding eigenvectors are:

$$
\left(-\frac{1}{B_{2}}, 1\right), \quad \text { and } \quad\left(\frac{2 \gamma_{2}\left(A_{1}-A_{2}-\beta_{1}+\gamma_{2}\right)}{B_{2}\left(-A_{1}-A_{2}+\beta_{1}+\gamma_{2}\right)\left(A_{1}-A_{2}+\beta_{1}+\gamma_{2}\right)}, 1\right) .
$$

Proof. The value of the Jacobian matrix of $T$ at the equilibrium point $E=(\bar{x}, \bar{y})$ is

$$
J_{T}(\bar{x}, \bar{y})=\left(\begin{array}{cc}
\frac{2 \beta_{1}}{\gamma_{2}-A_{2}+\beta_{1}+A_{1}} & \frac{\gamma_{2}-A_{2}+A_{1}-\beta_{1}}{B_{3}\left(A_{1}+\gamma_{2}-A_{2}+\beta_{1}\right)} \\
-\frac{B_{2}\left(\gamma_{2}-A_{2}+\beta_{1}-A_{1}\right)}{2 \gamma_{2}} & \frac{A_{2}+\gamma_{2}+A_{1}-\beta_{1}}{2 \gamma_{2}}
\end{array}\right) .
$$

The characteristic equation is given by

$$
\begin{gathered}
\lambda^{2}-\lambda\left(\frac{2 \beta_{1}}{\gamma_{2}-A_{2}+\beta_{1}+A_{1}}+\frac{A_{2}+\gamma_{2}+A_{1}-\beta_{1}}{2 \gamma_{2}}\right)+\frac{\beta_{1}\left(A_{2}+\gamma_{2}+A_{1}-\beta_{1}\right)}{\gamma_{2}\left(\gamma_{2}-A_{2}+\beta_{1}+A_{1}\right)} \\
-\frac{\left(\gamma_{2}-A_{2}+\beta_{1}-A_{1}\right)\left(\gamma_{2}-A_{2}+A_{1}-\beta_{1}\right)}{2 \gamma_{2}\left(A_{1}+\gamma_{2}-A_{2}+\beta_{1}\right)}=0 .
\end{gathered}
$$


Solutions of Equation (41) are:

$$
\lambda_{1}=1 \quad \text { and } \quad \lambda_{2}=\frac{A_{1}^{2}-A_{2}^{2}+2 A_{2} \beta_{1}-\beta_{1}^{2}+2 A_{2} \gamma_{2}+2 \beta_{1} \gamma_{2}-\gamma_{2}^{2}}{2 \gamma_{2}\left(A_{1}-A_{2}+\beta_{1}+\gamma_{2}\right)} .
$$

By using (40), we obtain the corresponding eigenvectors.

\section{Global behavior}

Theorem 18 Table 2 describes the global behavior of System (1).

Proof. Throughout the proof of theorem $\preccurlyeq$ will denote $\preccurlyeq_{s e}$.

$\left(\mathcal{R}_{i}, i=\overline{1,4}\right)$ By Theorem $9, E_{1}$ is locally asymptotically stable. Consider $M(t)=(0, t)$ for $t \geq \gamma_{2}-A_{2}$. Since $M(t)-T(M(t))=\left(-\frac{\alpha_{1}}{t+A_{1}}, \frac{t\left(t+A_{2}-\gamma_{2}\right)}{t+A_{2}}\right)$, we have $M(t) \leqslant T(M(t))$ for $t \geq \gamma_{2}-A_{2}$. By induction, $\left.T^{n} M(t) \leqslant T^{n+1}(M(t))\right) \leqslant E_{1}$ for all $n=0,1,2, \ldots$ because $M(t) \preccurlyeq$ $E_{1}$ for all $t \geq 0$. By monotonicity and boundedness, the sequence $\left\{T^{n}(M(t))\right\}$ has to converge to the unique equilibrium $E_{1}$. Consider $N(u)=(u, 0)$ for $u \geq 0$. Lemma 3 implies $T^{n}(N(u)) \rightarrow E_{1}$ as $n \rightarrow \infty$. Take any point $(x, y) \in[0,+\infty) \times[0,+\infty)$. Then there exists $t^{*}, u^{*} \geq 0$, such that $M\left(t^{*}\right) \leqslant(x, y) \leqslant(x, y) \leqslant N\left(u^{*}\right)$. The monotonicity of the map $T$ implies $\left.T^{n} M\left(t^{*}\right)\right) \leqslant T^{n}((x, y)) \leqslant T^{n}\left(N\left(u^{*}\right)\right)$. Since $\left.T^{n} M\left(t^{*}\right)\right), T^{n}\left(N\left(u^{*}\right)\right) \rightarrow E_{1}$ as $n \rightarrow$ $\infty$, then $T^{n}((x, y)) \rightarrow E_{1}$. This completes the proof.

$\left(\mathscr{R}_{5}\right)$ The first part of this theorem is proven in Theorem 9. The rest of the proof is similar to the proof of part $\left(\left(\mathcal{R}_{i}, i=\overline{1,4}\right)\right)$.

$\left(\mathscr{R}_{6}\right)$ By Lemma $3 y_{0}=0$ implies $y_{n}=0, \forall n \in \mathbb{N}$, and $x_{n} \rightarrow \frac{\alpha_{1}}{A_{1}-\beta_{1}}, n \rightarrow \infty$, which shows that $x$-axis is a subset of the basin of attraction of $E_{1}$.

Furthermore, every solution of (1) enters and stays in the box $\mathcal{B}\left(E_{2}\right)$ and so we can restrict our attention to solutions that starts in $\mathcal{B}\left(E_{2}\right)$. Clearly, the set $Q_{2}\left(E_{2}\right) \cap \mathcal{B}\left(E_{2}\right)$ is an invariant set with a single equilibrium point $E_{2}$ and by Theorem 3 , every solution that starts there is attracted to $E_{2}$. In view of Corollary 1, the interior of rectangle $\llbracket E_{2}, E_{1} \rrbracket$ is attracted to either $E_{1}$ or $E_{2}$, and because $E_{2}$ is the local attractor, it is attracted to $E_{2}$. If $(x, y) \in \mathcal{A}=\mathcal{B} \backslash\left(\llbracket E_{2}, E_{1} \rrbracket \cup\left(Q_{2}\left(E_{2}\right) \cap \mathcal{B}\right) \cup\{(x, 0): x \geq 0\}\right)$, then there exist the points $\left(x_{u}, y_{u}\right) \in A \cap Q_{4}\left(E_{2}\right)$ and $\left(x_{l}, y_{l}\right) \in Q_{2}\left(E_{2}\right) \cap \mathcal{B}$ such that $\left(x_{l}, y_{l}\right)$ $\preccurlyeq_{s e}(x, y) \preccurlyeq_{s e}\left(x_{u}, y_{u}\right)$. Consequently, $T^{n}\left(\left(x_{l}, y_{l}\right)\right) \preccurlyeq_{s e} T^{n}((x, y)) \preccurlyeq_{s e} T^{n}\left(\left(x_{u}, y_{u}\right)\right)$ for all $n$ $=1,2, \ldots$ and so $T^{n}((x, y)) \rightarrow E_{2}$ as $n \rightarrow \infty$, which completes the proof.

$\left(\mathscr{R}_{7}\right)$ The first part of this Theorem is proven in Theorem 13.

Now, we prove a global result.

$$
J_{T}\left(E_{1}\right)=\left(\begin{array}{ll}
\frac{\beta_{1}}{A_{1}} & -\frac{\alpha_{1}}{A_{1}\left(A_{1}-\beta_{1}\right)} \\
0 & \frac{\left(A_{1}-\beta_{1}\right) \gamma_{2}}{A_{1} A_{2}-\beta_{1} A_{2}+B_{2} \alpha_{1}}
\end{array}\right)
$$

The eigenvalues of $J_{T}\left(E_{1}\right)$ are given by $\lambda_{1}=\frac{\beta_{1}}{A_{1}}$ and $\lambda_{2}=\frac{\left(A_{1}-\beta_{1}\right) \gamma_{2}}{A_{1} A_{2}-\beta_{1} A_{2}+B_{2} \alpha_{1}}$ and so

$$
A_{2}<\gamma_{2}, \quad \alpha_{1}<\frac{\left(A_{1}-\beta_{1}\right)\left(\gamma_{2}-A_{2}\right)}{B_{2}} \Rightarrow \lambda_{2}>1, \quad A_{1}>\beta_{1} \Rightarrow \lambda_{1}<1 .
$$

The eigenvector of $T$ at $E_{1}$ that corresponds to the eigenvalue $\lambda_{1}<1$ is $(1,0)$.

The rest of the proof is similar to the proof of part $\left(\mathscr{R}_{6}\right)$.

$\left(\mathscr{R}_{8}, \mathscr{R}_{9}\right)$ The first part of theorem follows from Theorems 15 and 16. If parameters $\alpha_{1} \beta_{1}, A_{1}, \gamma_{2}, A_{2}$ and $B_{2}$ do not satisfy the condition (8) of Lemma 1, then the map $T$ defined on the set $\mathcal{R}=\mathbb{R}_{+}^{2}$, satisfies all conditions of Theorems 4, 6-8. This implies that 
Table 2 Global behavior of System (1)

\begin{tabular}{|c|c|}
\hline Region & Global behavior \\
\hline $\begin{array}{l}\mathcal{R}_{1} A_{1}>\beta_{1}, A_{2}<\gamma_{2}<A_{1}+A_{2}-\beta_{1}, \\
\text { or } \frac{\left(A_{1}-\beta_{1}\right)\left(\gamma_{2}-A_{2}\right)}{B_{2}}<\alpha_{1} \leq \frac{\left(A_{1}-A_{2}-\beta_{1}+\gamma_{2}\right)^{2}}{4 B_{2}} \\
\mathcal{R}_{2} A_{1}>\beta_{1}, A_{2}>\gamma_{2}, \alpha_{1} \leq \frac{\left(A_{1}-A_{2}-\beta_{1}+\gamma_{2}\right)^{2}}{4 B_{2}}, \\
\qquad A_{1}+\gamma_{2} \neq A_{2}+\beta_{1}, \\
\text { or } \\
\mathcal{R}_{3} \quad A_{1}>\beta_{1}, A_{2}=\gamma_{2}, \alpha_{1} \leq \frac{\left(A_{1}-A_{2}-\beta_{1}+\gamma_{2}\right)^{2}}{4 B_{2}} \\
\text { or } \\
\mathcal{R}_{4} \quad A_{1}>\beta_{1}, \alpha_{1}>\frac{\left(A_{1}-A_{2}-\beta_{1}+\gamma_{2}\right)^{2}}{4 B_{2}}\end{array}$ & $\begin{array}{l}\text { There exists a unique } \\
\text { equilibrium } E_{1} \text {, and it is globally } \\
\text { asymptotically stable (G.A.S.). } \\
\text { The basin of attraction of } E_{1} \text { is } \\
\text { given by } \mathcal{B}_{1}\left(E_{1}\right)=[0, \infty)^{2}\end{array}$ \\
\hline $\mathcal{R}_{5} \quad A_{1}>\beta_{1}, \gamma_{2}+\beta_{1} \leq A_{1}+A_{2}, \alpha_{1}=\frac{\left(A_{1}-\beta_{1}\right)\left(\gamma_{2}-A_{2}\right)}{B_{2}}$ & $\begin{array}{l}\text { There exists a unique } \\
\text { equilibrium } E_{1}=E_{2} \text { which is } \\
\text { non-hyperbolic. Furthermore, } \\
\text { this equilibrium is the global } \\
\text { attractor. Its basin of attraction is } \\
\text { given by } \mathcal{B}\left(E_{1}\right)=[0,+\infty)^{2} \text {. This } \\
\text { is an example of globally } \\
\text { attractive non-hyperbolic } \\
\text { equilibrium point }\end{array}$ \\
\hline $\mathcal{R}_{6} \quad A_{1}>\beta_{1}, A_{1}+A_{2}<\beta_{1}+\gamma_{2}, \alpha_{1}=\frac{\left(A_{1}-\beta_{1}\right)\left(\gamma_{2}-A_{2}\right)}{B_{2}}$ & $\begin{array}{l}\text { There exist two equilibrium } \\
\text { points } E=E_{1}=E_{3} \text { which is non- } \\
\text { hyperbolic, and } E_{2} \text {, which is } \\
\text { locally asymptotically stable. } \\
\text { Furthermore, the } x \text {-axis is the } \\
\text { basin of attraction of } E_{1} \text {. The } \\
\text { equilibrium point } E_{2} \text { is globally } \\
\text { asymptotically stable with the } \\
\text { basin of attraction } \mathcal{B}\left(E_{2}\right)=[0 \text {, } \\
+\infty) \times[0,+\infty)\end{array}$ \\
\hline $\mathcal{R}_{7} \quad A_{2}>\beta_{1}, A_{2}<\gamma_{2}, \alpha_{1}<\frac{\left(A_{1}-\beta_{1}\right)\left(\gamma_{2}-A_{2}\right)}{B_{2}}$ & $\begin{array}{l}\text { There exist two equilibrium } \\
\text { points } E_{1} \text {, which is a saddle, and } \\
E_{2} \text {, which is a locally } \\
\text { asymptotically stable equilibrium } \\
\text { point. Furthermore, the } x \text {-axis is } \\
\text { the global stable manifold of } \\
\mathcal{W}^{5}\left(E_{1}\right) \text {. The equilibrium point } E_{2} \\
\text { is globally asymptotically stable } \\
\text { with the basin of attraction } \\
\mathcal{B}\left(E_{2}\right)=[0,+\infty) \times[0,+\infty)\end{array}$ \\
\hline $\mathcal{R}_{8} \quad A_{1}<\beta_{1}, A_{1}+\gamma_{2}>A_{2}+\beta_{1}, \alpha_{1}<\frac{\left(A_{1}-A_{2}-p_{1}+\gamma_{2}\right)}{4 B_{2}}$ & $\begin{array}{l}\text { There exist two equilibrium } \\
\text { points } E_{3} \text {, which is a saddle, and } \\
E_{2} \text {, which is locally } \\
\text { asymptotically stable. } \\
\text { Furthermore, there exists the } \\
\text { global stable manifold } \mathcal{B}^{5}\left(E_{3}\right) \\
\text { that separates the positive } \\
\text { quadrant so that all orbits } \\
\text { below this manifold are } \\
\text { asymptotic to }(+\infty, 0) \text {, and all } \\
\text { orbits above this manifold are } \\
\text { asymptotic to the equilibrium } \\
\text { point } E_{2} \text {. All orbits that starts } \\
\text { on } \mathcal{B}^{5}\left(E_{3}\right) \text { are attracted to } E_{3}\end{array}$ \\
\hline
\end{tabular}


Table 2 Global behavior of System (1) (Continued)

\begin{tabular}{|c|c|}
\hline \multicolumn{2}{|l|}{ or } \\
\hline \multicolumn{2}{|l|}{$\begin{aligned} \mathcal{R}_{9} A_{1} & =\beta_{1}, A_{1}+A_{2}<\beta_{1}+\gamma_{2} \\
\alpha_{1} & <\frac{\left(A_{1}-A_{2}-\beta_{1}+\gamma_{2}\right)^{2}}{}\end{aligned}$} \\
\hline$\frac{\left(A_{1}-\beta_{1}\right)\left(\gamma_{2}-A_{2}\right)}{B_{2}}<\alpha_{1}<\frac{\left(A_{1}-A_{2}-\beta_{1}+\gamma_{2}\right)^{2}}{4 B_{2}}$ & $\begin{array}{l}\text { There exist three equilibrium } \\
\text { points } E_{1}, E_{2} \text {, and } E_{3} \text {, where } E_{1} \\
\text { and } E_{2} \text { are locally asymptotically } \\
\text { stable and } E_{3} \text { is a saddle. There } \\
\text { exists the global stable manifold } \\
\mathcal{W}^{5}\left(E_{3}\right) \text { that separates the } \\
\text { positive quadrant so that all } \\
\text { orbits below this manifold are } \\
\text { attracted to the equilibrium } \\
\text { point } E_{1} \text {, and all orbits above } \\
\text { this manifold are attracted to } \\
\text { the equilibrium point } E_{2} \text {. All } \\
\text { orbits that starts on } \mathcal{W}^{5}\left(E_{3}\right) \text { are } \\
\text { attracted to } E_{3} \text {. The global } \\
\text { unstable manifold } \mathcal{W}^{5}\left(E_{3}\right) \text { is the } \\
\text { graph of a continuous strictly } \\
\text { decreasing function, and } \mathcal{W}^{u}\left(E_{3}\right) \\
\text { has endpoints } E_{2} \text { and } E_{1}\end{array}$ \\
\hline $\mathcal{R}_{11} \quad A_{1}>\beta_{1}, A_{1}+A_{2}<\beta_{1}+\gamma_{2}, \alpha_{1}=\frac{\left(A_{1}-A_{2}-\beta_{1}+\gamma_{2}\right)^{2}}{4 B_{2}}$ & $\begin{array}{l}\text { There exist two equilibrium } \\
\text { points } E=E_{2}=E_{3} \text { and } E_{1} \text {. } E_{1} \text { is } \\
\text { locally asymptotically stable and } \\
E \text { is non-hyperbolic. There exists } \\
\text { a continuous increasing curve } \\
\mathcal{W}_{E} \text { which is a subset of the } \\
\text { basin of attraction of } E \text {. All orbits } \\
\text { that start below this curve are } \\
\text { attracted to } E_{1} \text {. All orbits that } \\
\text { start above this curve are } \\
\text { attracted to } E\end{array}$ \\
\hline $\mathcal{R}_{12} \quad A_{1}<\beta_{1}, A_{1}+\gamma_{2}>A_{2}+\beta_{1}, \alpha_{1}=\frac{\left(A_{1}-A_{2}-\beta_{1}+\gamma_{2}\right)^{2}}{4 B_{2}}$ & $\begin{array}{l}\text { There exists a unique } \\
\text { equilibrium point } E=E_{2}=E_{3} \\
\text { which is non-hyperbolic. There } \\
\text { exists a continuous increasing } \\
\text { curve } \mathcal{W}_{E} \text { which is a subset of } \\
\text { basin of attraction of } E \text {. All orbits } \\
\text { that start below this curve are } \\
\text { attracted to }(+\infty, 0) \text {. All orbits } \\
\text { that start above this curve are } \\
\text { attracted to } E \text {. This is an } \\
\text { example of semi-stable non- } \\
\text { hyperbolic equilibrium point }\end{array}$ \\
\hline $\begin{aligned} \mathcal{R}_{13} A_{1} & =\beta_{1}, A_{1}+A_{2}<\beta_{1}+\gamma_{2}, \\
\alpha_{1} & =\frac{\left(A_{1}-A_{2}-\beta_{1}+\gamma_{2}\right)^{2}}{4 B_{2}} \\
\mathcal{R}_{14} A_{1} & <\beta_{1}, A_{2}<\gamma_{2}<-A_{1}+A_{2}+\beta_{1},\end{aligned}$ & \\
\hline$\alpha_{1} \leq \frac{\left(A_{1}-A_{2}-\beta_{1}+\gamma_{2}\right)^{2}}{4 B_{2}}$ & $\begin{array}{l}\text { System (1) does not posses an } \\
\text { equilibrium point. Its behavior is } \\
\text { as follows } x_{n} \rightarrow \infty, y_{n} \rightarrow \infty, n \\
\rightarrow \infty\end{array}$ \\
\hline $\begin{array}{l}\mathcal{R}_{15} A_{1}<\beta_{1}, A_{2} \geq \gamma_{2}, \\
\quad \alpha_{1} \leq \frac{\left(A_{1}-A_{2}-\beta_{1}+\gamma_{2}\right)^{2}}{4 B_{2}} \\
\text { or } \\
\mathcal{R}_{16} \quad A_{1} \leq \beta_{1}, \alpha_{1}>\frac{\left(A_{1}-A_{2}-\beta_{1}+\gamma_{2}\right)^{2}}{4 B_{2}}\end{array}$ & \\
\hline $\begin{aligned} \mathcal{R}_{\text {Or }} & A_{17}=\beta_{1}, A_{1}+A_{2}>\gamma_{2}+\beta_{1} \\
\alpha_{1} & \leq \frac{\left(A_{1}-A_{2}-\beta_{1}+\gamma_{2}\right)^{2}}{4 B_{2}}\end{aligned}$ & \\
\hline
\end{tabular}


there exists the global stable manifold $\mathcal{W}^{s}\left(E_{3}\right)$ that separates the first quadrant into two invariant regions $\mathcal{W}^{-}\left(E_{3}\right)$ (above the stable manifold) and $\mathcal{W}^{+}\left(E_{3}\right)$ (below the stable manifold) which are connected. Now, we show that each orbit starting in the region $\mathcal{W}^{+}\left(E_{3}\right)$ is asymptotic to $(\infty, 0)$. Indeed, set $T_{1}(x, y)=\frac{\alpha_{1}+\beta_{1} x}{A_{1}+y}, T_{2}(x, y)=\frac{\gamma_{2} y}{A_{2}+B_{2} x+y}$. Take $x=$ $\left(x_{0}, y_{0}\right) \in \mathcal{W}^{+}\left(E_{3}\right) \cap \mathscr{R}(+,-)$, where $\mathscr{R}(+,-)=\left\{(x, y) \in \mathscr{R}: T_{1}(x, y)>x, T_{2}(x, y)<y\right\}$. As is known, see [12], the set $\mathscr{R}(+,-)$ is invariant. We have

$$
T_{1}\left(x_{0}, y_{0}\right)=\frac{\alpha_{1}+\beta_{1} x_{0}}{A_{1}+y_{0}}>x_{0}, \quad T_{2}\left(x_{0}, y_{0}\right)=\frac{\gamma_{2} y_{0}}{A_{2}+B_{2} x_{0}+y_{0}}<y_{0},
$$

which implies the following

$$
\left(x_{0}, y_{0}\right) \preccurlyeq_{s e}\left(T_{1}\left(x_{0}, y_{0}\right), T_{2}\left(x_{0}, y_{0}\right)\right) \Leftrightarrow\left(x_{0}, y_{0}\right) \preccurlyeq_{s e} T\left(x_{0}, y_{0}\right) .
$$

By monotonicity, $T\left(x_{0}, y_{0}\right) \leqslant s e T^{2}\left(x_{0}, y_{0}\right)$ and by induction, $T^{n}\left(x_{0}, y_{0}\right) \leqslant s_{s e} T^{n+1}\left(x_{0}\right.$, $\left.y_{0}\right)$. This implies that sequence $\left\{x_{n}\right\}$ is non-decreasing and $\left\{y_{n}\right\}$ is non-increasing. Since, $\left\{y_{n}\right\}$ is bounded from above, hence it must converges. Now $\lim _{n \rightarrow \infty} \mathrm{y}_{n}=0$ since otherwise $\left(x_{n}, y_{n}\right)$ will converge to another limit which is strictly south-east of $E_{3}$, which is impossible. By Lemma 3, $x_{n} \rightarrow \infty$. By Theorems 6-8 for all $(x, y) \in \mathcal{W}^{+}\left(E_{3}\right)$, there exists $n_{0}>0$ such that $T^{n}((x, y)) \in \operatorname{int}\left(\mathcal{Q}_{4}\left(E_{3}\right) \cap \mathscr{R}\right), n>n_{0}$. We see that for all $(x, y) \in \operatorname{int}\left(Q_{4}\right.$ $\left.\left.\left(E_{3}\right)\right) \cap \mathscr{R}\right)$, there exists $\left(x_{l}, y_{l}\right) \in \mathcal{W}^{+}\left(E_{3}\right) \cap \mathscr{R}(+,-)$ such that $\left(x_{l}, y_{l}\right) \leqslant(x, y)$. By monotonicity $T^{n}\left(\left(x_{l}, y_{l}\right)\right) \leqslant T^{n}((x, y)) \leqslant(\infty, 0)$. This implies $T^{n}((x, y)) \rightarrow(\infty, 0)$ as $n \rightarrow \infty$.

Now, we show that each orbit starting in the region $\mathcal{W}^{-}\left(E_{3}\right)$ converges to $E_{2}$. By Theorem 6, for all $(x, y) \in \mathcal{W}^{-}\left(E_{3}\right)$, there exists $n_{0}>0$ such that, $T^{n}((x, y)) \in \operatorname{int}\left(Q_{2}\left(E_{3}\right) \cap\right.$ $\mathscr{R}), n>n_{0}$. Set $M(t)=(0, t)$ By part $\left(\left(\mathcal{R}_{i}, i=\overline{1,4}\right)\right)$, for $\mathrm{t} \geq \gamma_{2}-A_{2}$, we have $M(t) \preccurlyeq T(M(t)) \preccurlyeq E_{2}$.. By using monotonicity, $T^{n}(M(t)) \rightarrow E_{2}$ as $\mathrm{n} \rightarrow \infty$. By Corollary 1 , the interior of rectangle $\llbracket E_{2}, E_{3} \rrbracket$ is attracted to either $E_{2}$ or $E_{3}$, and because $E_{2}$ is local attractor, it is attracted to $E_{2}$. If $(x, y) \in \operatorname{int}\left(Q_{2}\left(E_{3}\right) \cap \mathscr{R}\right)$, then there exist the points $\left(x_{r}, y_{r}\right) \in \llbracket E_{2}, E_{3} \rrbracket$ and $t^{*} \geq \gamma_{2}-A_{2}$, such that $M\left(t^{*}\right) \preccurlyeq_{s e}(x, y) \preccurlyeq_{s e}\left(x_{r}, y_{r}\right)$. Consequently, $T^{n}\left(M\left(t^{*}\right)\right) \preccurlyeq_{s e} T^{n}((x, y)) \preccurlyeq_{s e} T^{n}\left(\left(x_{r}, y_{r}\right)\right)$ for all $n=1,2, \ldots$ and so $T^{n}((x, y)) \rightarrow$ $E_{2}$ as $n \rightarrow \infty$.

Now, assume that parameters $\alpha_{1}, \beta_{1}, A_{1}, \gamma_{2}, A_{2}$, and $B_{2}$ satisfy the condition (8) and inequality 1. i) of Lemma 1 . Then the set

$$
\mathcal{I}=\left\{\left(x, \frac{A_{1} A_{2} \beta_{1}+x A_{1} B_{2} \beta_{1}}{B_{2} \alpha_{1}-A_{2} \beta_{1}}\right): x \geq 0\right\}
$$

is invariant and contains the equilibrium point $E_{3}$, and $T(x, y)=E_{3}$ for $(x, y) \in \mathscr{Z}$. In view of the uniqueness of global stable manifold, we conclude that $\mathcal{W}^{s}\left(E_{3}\right)=\mathscr{g}$. Take any point $(x, y) \in \mathcal{W}^{+}\left(E_{3}\right)$. Then there exists the point $\left(x_{l}, y_{l}\right) \in \mathscr{J}$ such that $\left(x_{l}, y_{l}\right)$ $<<_{s e}(x, y)$. Since, the map $T$ is strongly competitive, then $E_{3}=T\left(x_{l}, y_{l}\right)<<_{s e} T(x, y)$. This implies $T(x, y) \in \operatorname{int}\left(Q_{4}\left(E_{3}\right) \cap \mathscr{R}\right)$. Similarly, if $(x, y) \in \mathcal{W}^{-}\left(E_{3}\right)$, then $T(x, y) \in$ int $\left(Q_{2}\left(E_{3}\right) \cap \mathscr{R}\right)$. The rest of the proof is similar to the proof of the first case. This completes the proof.

$\left(\mathscr{R}_{10}\right)$ The first part of the theorem follows from Theorem 10. If parameters $\alpha_{1}, \beta_{1}$, $A_{1}, \gamma_{2}, A_{2}$, and $B_{2}$ do not satisfy the condition (8) of Lemma 1 , then the map $T$, defined on the set $\mathcal{R}=\mathbb{R}_{+}^{2}$, satisfies all conditions of Theorems 4, 6-8. This implies that there exists the global stable manifold $\mathcal{W}^{s}\left(E_{3}\right)$ that separates the first quadrant into two 
invariant regions $\mathcal{W}^{+}\left(E_{3}\right)$ (below the stable manifold) and $\mathcal{W}^{-}\left(E_{3}\right)$ (above the stable manifold) which are connected.

Using Theorems 6, 7, and 8, we have that for all $(x, y) \in \mathcal{W}^{+}\left(E_{3}\right)$, there exists $n_{0}>0$ such that for $n>n_{0}, T^{n}((x, y)) \in \operatorname{int}\left(Q_{4}\left(E_{3}\right) \cap \mathscr{R}\right)$, and for all $(x, y) \in \mathcal{W}^{-}\left(E_{3}\right)$, there exists $n_{1}>0$ such that for all $n>n_{1}, T^{n}((x, y)) \in \operatorname{int}\left(Q_{2}\left(E_{3}\right) \cap \mathscr{R}\right)$. Now, we show that each orbit starting in the region $\operatorname{int}\left(Q_{4}\left(E_{3}\right)\right)$ converges to $E_{1}$, and each orbit starting in the region $\operatorname{int}\left(Q_{2}\left(E_{3}\right)\right)$ converges to $E_{2}$.

Take $0 \leq t \leq\left(\gamma_{2}-A_{2}\right) / B_{2}, U(t)=\left(t,-A_{2}-t B_{2}+\gamma_{2}\right)$. It is easy to see that the following holds

$$
\begin{aligned}
& U(\bar{x})=E=E_{2}=E_{3} \preccurlyeq E_{1} \quad \text { where } \bar{x}=x_{2}=x_{3} \quad \text { and } \\
& U(t)-T(U(t))=\left(-\frac{\left(-A_{1}+A_{2}+2 t B_{2}+\beta_{1}-\gamma_{2}\right)^{2}}{4 B_{2}\left(A_{1}-A_{2}-t B_{2}+\gamma_{2}\right)}, 0\right) .
\end{aligned}
$$

Since $x_{2}$ and $x_{3}$ are solutions of the equation $B_{2} t^{2}+\left(-A_{1}+A_{2}+\beta_{1}-\gamma_{2}\right) t+\alpha_{1}=0$ and the following inequality holds $A_{2}+t B_{2}-\gamma_{2}<0$, we have that $U(t) \preccurlyeq_{s e} T(U(t))$ for 0 $\leq t \leq x_{2}$ and $x_{3} \leq t \leq\left(\gamma_{2}-A_{2}\right) / B_{2}$ and $\left.T(U(t))\right) \preccurlyeq_{s e} U(t)$ for $x_{2} \leq t \leq x_{3}$.

By using monotonicity of $T$, we have that for $0 \leq t<x_{2}, T^{n}(U(t)) \leqslant T^{n+1}(U(t)) \leqslant E_{2}$, and for $x_{2} \leq t<x_{3}, E_{2} \leqslant T^{n+1}(U(t)) \leqslant T^{n}(U(t)) \leqslant E_{3}$. This implies $T^{n}(U(t)) \rightarrow E_{2}$ as $n$ $\rightarrow \infty$. Similarly, for $x_{3} \leq t \leq\left(\gamma_{2}-A_{2}\right) / B_{2}$, we have $E_{3} \leqslant T^{n}(U(t)) \leqslant T^{n+1}(U(t)) \leqslant E_{1}$. This implies $T^{n}(U(t)) \rightarrow E_{1}$ as $n \rightarrow \infty$. By using the property of points $U(t)$ and $N(u)$, we have that for each point $(x, y) \in \operatorname{int}\left(Q_{4}\left(E_{3}\right) \cap \mathscr{R}\right)$, there exits $x_{3}<t^{*}<\left(\gamma_{2}-A_{2}\right) / B_{2}$ and $u^{*}>0$ such that $U\left(t^{*}\right) \leqslant(x, y) \leqslant N\left(u^{*}\right)$. By using monotonicity of $T$, we have $T^{n}\left(U\left(t^{*}\right)\right)$ $\left.\leqslant T^{n}((x, y)) \leqslant T^{n}\left(N\left(u^{*}\right)\right)\right)$. This implies $T^{n}((x, y)) \rightarrow E_{1}$ as $n \rightarrow \infty$. Furthermore, for each point $(x, y) \in \operatorname{int}\left(Q_{2}\left(E_{3}\right) \cap \mathscr{R}\right)$, there exist $t_{1}>0$ and $t_{2}, x_{2}<t_{2}<x_{3}$ such that $M$ $\left(t_{1}\right) \leqslant(x, y) \leqslant U\left(t_{2}\right)$. By using monotonicity of $T$, we have $T^{n}\left(M\left(t_{1}\right)\right) \leqslant T^{n}((x, y)) \leqslant T^{n}$ $\left(U\left(t_{2}\right)\right)$. This implies $T^{n}((x, y)) \rightarrow E_{2}$ as $n \rightarrow \infty$.

Now, assume that parameters $\alpha_{1}, A_{1}, \gamma_{2}, A_{2}$, and $B_{2}$ satisfy the condition (8) and inequality $1 . i)$ of Lemma 1 . Then the set

$$
\mathcal{I}=\left\{\left(x, \frac{A_{1} A_{2} \beta_{1}+x A_{1} B_{2} \beta_{1}}{B_{2} \alpha_{1}-A_{2} \beta_{1}}\right): x \geq 0\right\}
$$

is invariant and contains the equilibrium point $E_{3}$ and $T(x, y)=E_{3}$ for $(x, y) \in I$. In view of the uniqueness of global stable manifold, we conclude that $\mathcal{W}^{s}\left(E_{3}\right)=\mathscr{Z}$. Take any point $(x, y) \in \mathcal{W}^{+}\left(E_{3}\right)$, then there exists the point $\left(x_{l}, y_{l}\right) \in \mathscr{Z}$ such that $\left(x_{l}, y_{l}\right)<<_{\text {se }}$ $(x, y)$. Since, the map $T$ is strongly competitive, then $E_{3}=T\left(x_{l}, y_{l}\right)<<_{s e} T(x, y)$. This implies $T(x, y) \in \operatorname{int}\left(Q_{4}\left(E_{3}\right) \cap \mathscr{R}\right)$. Similarly, if $(x, y) \in \mathcal{W}^{-}\left(E_{3}\right)$, then $T(x, y) \in \operatorname{int}\left(Q_{2}\right.$ $\left.\left(E_{3}\right) \cap \mathscr{R}\right)$. The rest of the proof is similar to the proof of the first case. This completes the proof.

$\left(\mathscr{R}_{11}\right)$ The first part of theorem follows from Theorems 15 and 16. If parameters $\alpha_{1}$, $\beta_{1}, A_{1}, \gamma_{2}, A_{2}$, and $B_{2}$ do not satisfy the condition (8) of Lemma 1 , then the map $T$, defined on the set $\mathcal{R}=\mathbb{R}_{+}^{2}$, satisfies all conditions of Theorems 4,6 , and 8 . This implies that there exists an invariant curve $\mathcal{C}$, which is a subset of the basin of attraction of the equilibrium point $E$, which separates the first quadrant into two invariant regions, $\mathcal{W}^{+}(E)$ (below the stable manifold) and $\mathcal{W}^{-}(E)$ (above the stable manifold) which are connected. 
By Theorems 6 and 7 and 8 for all $(x, y) \in \mathcal{W}^{+}(E)$, there exists $n_{0}>0$ such that $T^{n}$ $((x, y)) \in \operatorname{int}\left(Q_{4}(E) \cap \mathscr{R}\right)$ for $n>n_{0}$. For all $(x, y) \in \mathcal{W}^{-}(E)$, there exists $n_{1}>0$ such that for all $n>n_{1}, T^{n}((x, y)) \in \operatorname{int}\left(Q_{2}(E) \cap \mathscr{R}\right)$. Now, we show that each orbit starting in the region $\operatorname{int}\left(Q_{4}(E)\right)$ converges to $E_{1}$, and each orbit starting in the region $\operatorname{int}\left(Q_{2}(E)\right)$ converges $E$.

Now, for $0 \leq t \leq\left(\gamma_{2}-A_{2}\right) / B_{2}$, take $U(t)=\left(t,-A_{2}-t B_{2}+\gamma_{2}\right)$ Since $\alpha_{1}=\left(A_{1}-A_{2}-\beta_{1}+\right.$ $\left.\gamma^{2}\right)^{2} /\left(4 B_{2}\right)$, it is easy to see that the following holds

$$
\begin{aligned}
& U(\bar{x})=E=E_{2}=E_{3} \preccurlyeq E_{1} \quad \text { where } \bar{x}=x_{2}=x_{3} \quad \text { and } \\
& U(t)-T(U(t))=\left(-\frac{\left(-A_{1}+A_{2}+2 t B_{2}+\beta_{1}-\gamma_{2}\right)^{2}}{4 B_{2}\left(A_{1}-A_{2}-t B_{2}+\gamma_{2}\right)}, 0\right) .
\end{aligned}
$$

Since $A_{2}+t B_{2}-\gamma_{2}<0$, we have $U(t) \preccurlyeq_{s e} T(U(t)) \preccurlyeq$ for $0 \leq t \leq\left(\gamma_{2}-A_{2}\right) / B_{2}$.

By using monotonicity of $T$, we have that $T^{n}(U(t)) \preccurlyeq T^{n+1}(U(t)) \preccurlyeq E$ for $0 \leq t<\bar{x}$. This implies $T^{n}(U(t)) \rightarrow E$ as $n \rightarrow \infty$. Similarly, for $\bar{x} \leq t<\left(\gamma_{2}-A_{2}\right) / B_{2}$, $E \preccurlyeq T^{n}(U(t)) \preccurlyeq T^{n+1}(U(t)) \preccurlyeq E_{1}$. This implies $T^{n}(U(t)) \rightarrow E_{1}$ as $n \rightarrow \infty$. By using the property of the points $U(t)$ and $N(u)$, we have that for each point $(x, y) \in \operatorname{int}\left(Q_{4}(E) \cap\right.$ $\mathscr{R})$, there exist $\bar{x}<t^{*}<\left(\gamma_{2}-A_{2}\right) / B_{2}$ and $u^{*}>0$ such that $U\left(t^{*}\right) \preccurlyeq(x, y) \preccurlyeq N\left(u^{*}\right)$. By using monotonicity of $T$, we have that $\left.T^{n}\left(U\left(t^{*}\right)\right) \preccurlyeq T^{n}((x, y)) \preccurlyeq T^{n}\left(N\left(u^{*}\right)\right)\right)$. This implies $T^{n}((x, y)) \rightarrow E_{1}$ as $n \rightarrow \infty$. Furthermore, for each point $(x, y) \in \operatorname{int}\left(Q_{2}(E) \cap \mathscr{R}\right)$ there exists $t_{1}>0$ such that $M\left(t_{1}\right) \preccurlyeq(x, y) \preccurlyeq E$. By using monotonicity of $T$, we have $T^{n}\left(M\left(t_{1}\right)\right) \preccurlyeq T^{n}((x, y)) \preccurlyeq E$. This implies $T^{n}((x, y)) \rightarrow E$ as $n \rightarrow \infty$.

Now, assume that parameters $\alpha_{1}, \beta_{1}, A_{1}, \gamma_{2}, A_{2}$, and $B_{2}$ satisfy the condition (8) and inequality 1.i) of Lemma 1 . The proof of Theorem is similar to the proof of Theorem in the regions $\left(\mathscr{R}_{9}\right)$ and $\left(\mathscr{R}_{10}\right)$.

$\left(\mathscr{R}_{12}, \mathscr{R}_{13}\right)$ The first part of theorem follows from Theorems 15 and 17. If parameters $\alpha_{1}, \beta_{1}, A_{1} \gamma_{2}, A_{2}$, and $B_{2}$ do not satisfy (8) of Lemma 1, then the map $T$, defined on the set $\mathcal{R}=\mathbb{R}_{+}^{2}$, satisfies all conditions of Theorems 4,6 , and 8 . This implies that there exists an invariant curve $\mathcal{C}$, which is a subset of the basin of attraction of the equilibrium point $E$, and which separates the first quadrant into two invariant regions, $\mathcal{W}^{+}(E)$ (below the stable manifold) and $\mathcal{W}^{-}(E)$ (above the stable manifold) which are connected.

By Theorems 6 and 8 for all $(x, y) \in \mathcal{W}^{+}(E)$, there exists $n_{0}>0$ such that $T^{n}((x, y)) \in$ $\operatorname{int}\left(Q_{4}(E) \cap \mathscr{R}\right)$ for $n>n_{0}$, and for all $(x, y) \in \mathcal{C}^{-}(E)$, there exists $n_{1}>0$ such that $T^{n}((x$, $y)) \in \operatorname{int}\left(Q_{2}(E) \cap \mathscr{R}\right)$ for all $n>n_{1}$. Now, we show that each orbit starting in the region $\operatorname{int}\left(Q_{4}(E)\right)$ is asymptotic to $(\infty, 0)$ and each orbit starting in the region $\operatorname{int}\left(Q_{2}(E)\right)$ converges to $E$.

Since $\alpha_{1}=\left(A_{1}-A_{2} \beta_{1}+\gamma_{2}\right)^{2} /\left(4 B_{2}\right)$, for $0 \leq t \leq\left(\gamma_{2}-A_{2}\right) / B_{2}$, we have $U(t)=\left(t,-A_{2}\right.$ $\left.t B_{2}+\gamma_{2}\right)$. It is easy to see

$$
\begin{aligned}
& U(\bar{x})=E=E_{2}=E_{3} \quad \text { where } \bar{x}=x_{2}=x_{3} \quad \text { and } \\
& U(t)-T(U(t))=\left(-\frac{\left(-A_{1}+A_{2}+2 t B_{2}+\beta_{1}-\gamma_{2}\right)^{2}}{4 B_{2}\left(A_{1}-A_{2}-t B_{2}+\gamma_{2}\right)}, 0\right) .
\end{aligned}
$$

Since $A_{2}+t B_{2}-\gamma_{2}<0$, for $0 \leq t \leq\left(\gamma_{2}-A_{2}\right) / B_{2}$, we have $U(t) \preccurlyeq s e T(U(t))$.

By using monotonicity of $T$, we have $E \preccurlyeq T^{n}(U(t)) \preccurlyeq T^{n+1}(U(t)) \preccurlyeq E_{1} 0 \leq t<\bar{x}$. This implies $T^{n}(U(t)) \rightarrow E$ as $n \rightarrow \infty$. Similarly, $E \preccurlyeq T^{n}(U(t)) \preccurlyeq T^{n+1}(U(t)) \preccurlyeq(\infty, 0)$ for $\bar{x}<t^{*}<\left(\gamma_{2}-A_{2}\right) / B_{2}$. This implies $T^{n}(U(t)) \rightarrow(\infty, 0)$ as $n \rightarrow \infty$. For each point $(x, y)$ 
$\in \operatorname{int}\left(Q_{4}\left(E_{3}\right) \quad \cap \quad \mathscr{R}\right), \quad$ there exists $\bar{x}<t^{*}<\left(\gamma_{2}-A_{2}\right) / B_{2}$ such that $0 \leq t<\bar{x} .0 \leq t<\bar{x}$. By monotonicity of $T$, we have $T^{n}\left(U\left(t^{*}\right)\right) \preccurlyeq T^{n}((x, y)) \preccurlyeq(\infty, 0)$. This implies $T^{n}((x, y)) \rightarrow(\infty, 0)$ as $n \rightarrow \infty$. Furthermore, for each point $(x, y) \in \operatorname{int}\left(Q_{2}\right.$ $\left.\left(E_{3}\right) \cap \mathscr{R}\right)$, there exists $t_{1}>0$ such that $U\left(t^{*}\right) \preccurlyeq(x, y) \preccurlyeq N\left(u^{*}\right)$. By monotonicity of $T$, we have $T^{n}\left(M\left(t_{1}\right)\right) \preccurlyeq T^{n}((x, y)) \preccurlyeq E$. This implies $T^{n}((x, y)) \rightarrow E$ as $n \rightarrow \infty$.

If parameters $\alpha_{1}, \beta_{1}, A_{1}, \gamma_{2}, A_{2}$, and $B_{2}$ satisfy the condition (8) and inequality $\left.1 . \mathrm{i}\right)$ of Lemma 1 , then the proof of Theorem is similar to the proof of parts $\left(\mathscr{R}_{9}\right)$ and $\left(\mathscr{R}_{10}\right)$. This completes the proof of Theorem in the regions $\mathscr{R}_{12}, \mathscr{R}_{13}$. This is an example of semistable non-hyperbolic equilibrium point.

$\left(\mathcal{R}_{i}, i=\overline{14,17}\right)$ Assumptions of this theorem imply that equilibrium does not exist. Set $M(t)=(0, t)$ for $t \geq \gamma_{2}-A_{2}$. Since $M(t)-T(M(t))=\left(-\frac{\alpha_{1}}{t+A_{1}}, \frac{t\left(t+A_{2}-\gamma_{2}\right)}{t+A_{2}}\right)$, we have $M(t) \leqslant T(M(t))$ for $t \geq \gamma_{2}-A_{2}$. By using monotonicity $\left.T^{n}(M(t)) \leqslant T^{n+1}(M(t))\right)$, for all $n=0,1,2, \ldots$ Set $\left(x_{n^{\prime}}^{*} y_{n}^{*}\right)=T^{n}(M(t))$. This implies that $\left\{\gamma_{n}^{*}\right\}$ is non-increasing and bounded, hence it has to converge. Set $\lim _{n \rightarrow \infty} \gamma_{n}^{*}=\gamma^{*}$. Since $\left\{x_{n}^{*}\right\}$ is unbounded and non-decreasing, we have that $x_{n}^{*} \rightarrow \infty$. By using the second equation of the System (1), we see that $y^{*}=0$. Take any point $(x, y) \in[0, \infty)^{2}$. Then there exists $t^{*}$, such that $M$ $\left(t^{*}\right) \leqslant(x, y) \leqslant(\infty, 0)$. By using monotonicity, $T^{n}\left(M\left(t^{*}\right)\right) \leqslant\left(T^{n}((x, y)) \leqslant(\infty, 0)\right.$ as Since $T^{n}\left(M\left(t^{*}\right)\right) \rightarrow(\infty, 0)$ as $n \rightarrow \infty$, we obtain $T^{n}((x, y)) \rightarrow(\infty, 0)$ as $n \rightarrow \infty$, as which completes the proof of theorem.

\footnotetext{
Author details

${ }^{1}$ Department of Mathematics, University of Sarajevo, Sarajevo, Bosnia and Herzegovina ${ }^{2}$ Department of Mathematics, University of Rhode Island, Kingston, RI 02881-0816, USA
}

Authors' contributions

All authors contributed equally to the manuscript and read and approved the final draft.

Competing interests

The authors declare that they have no competing interests.

Received: 26 January 2011 Accepted: 23 August 2011 Published: 23 August 2011

References

1. Camouzis, E, Kulenović, MRS, Ladas, G, Merino, O: Rational systems in the plane. J Differ Equ Appl. 15, 303-323 (2009). doi:10.1080/10236190802125264

2. Kulenović, MRS, Merino, O: Invariant manifolds for competitive discrete systems in the plane. Int J Bifurcat Chaos. 20, 2471-2486 (2010). doi:10.1142/S0218127410027118

3. Kulenović, MRS, Merino, O: Global bifurcation for competitive systems in the plane. Discret Cont Dyn Syst B. 12, 133-149 (2009)

4. AlSharawi, Z, Rhouma, M: Coexistence and extinction in a competitive exclusion Leslie/Gower model with harvesting and stocking. J Differ Equ Appl. 15, 1031-1053 (2009). doi:10.1080/10236190802459861

5. Cushing, JM, Levarge, S, Chitnis, N, Henson, SM: Some discrete competition models and the competitive exclusion principle. J Differ Equ Appl. 10, 1139-1152 (2004). doi:10.1080/10236190410001652739

6. Kulenović, MRS, Nurkanović, M: Asymptotic behavior of a linear fractional system of difference equations. J Inequal Appl. 127-143 (2005)

7. Clark, D, Kulenović, MRS, Selgrade, JF: Global asymptotic behavior of a two dimensional difference equation modelling competition. Nonlinear Anal TMA. 52, 1765-1776 (2003). doi:10.1016/S0362-546X(02)00294-8

8. Hirsch, MW: Systems of differential equations which are competitive or cooperative. I Limits Sets SIAM J Math Anal. 13(2):167-179 (1982)

9. Hirsch, M, Smith, H: Monotone dynamical systems. In: Canada A, Drabek P, Fonda A (eds.) Handbook of Differential Equations: Ordinary Differential Equations, vol. II, pp. 239-357. Elsevier, Amsterdam (2005)

10. Kalabušić, S, Kulenović, MRS, Pilav, E: Global dynamics of a competitive system of rational difference equations in the plane. Adv Differ Equ 30 (2009). Article ID 132802

11. Kulenović, MRS, Merino, O: Discrete Dynamical Systems and Difference Equations with Mathematica. Chapman \& Hall/ CRC Press, Boca Raton (2002)

12. Kulenović, MRS, Merino, O: Competitive-exclusion versus competitive-coexistence for systems in the plane. Discret Cont Dyn Syst Ser B. 6, 1141-1156 (2006) 
13. Leonard, WJ, May, R: Nonlinear aspects of competition between species. SIAM J Appl Math. 29, 243-275 (1975). doi:10.1137/0129022

14. Smith, HL: Invariant curves for mappings. SIAM J Math Anal. 17, 1053-1067 (1986). doi:10.1137/0517075

15. Smith, HL: Periodic competitive differential equations and the discrete dynamics of competitive maps. J Differ Equ. 64 , 165-194 (1986). doi:10.1016/0022-0396(86)90086-0

16. Smith, HL: Periodic solutions of periodic competitive and cooperative systems. SIAM J Math Anal. 17, 1289-1318 (1986). doi:10.1137/0517091

17. Smith, HL: Planar competitive and cooperative difference equations. J Differ Equ Appl. 3, 335-357 (1998). doi:10.1080/ 10236199708808108

18. Smith, HL: Non-monotone systems decomposable into monotone systems with negative feedback. J Math Biol. 53, 747-758 (2006). doi:10.1007/s00285-006-0004-3

19. Takáč, P: Domains of attraction of generic $\omega$-limit sets for strongly monotone discrete-time semigroups. J Reine Angew Math. 423, 101-173 (1992)

20. de Mottoni, P, Schiaffino, A: Competition systems with periodic coefficients: a geometric approach. J Math Biol. 11, 319-335 (1981). doi:10.1007/BF00276900

21. Hess, P: Periodic-Parabolic Boundary Value Problems and Positivity. In Pitman Research Notes in Mathematics Series, vol. 247,Longman Scientific \& Technical, Harlow (1991)

22. Kocic, V, Ladas, G: Global Behavior of Nonlinear Difference Equations of Higher Order with Applications. Kluwer, Dordreht (1993)

doi:10.1186/1687-1847-2011-29

Cite this article as: Kalabušić et al:: Dynamics of a two-dimensional system of rational difference equations of Leslie-Gower type. Advances in Difference Equations 2011 2011:29.

\section{Submit your manuscript to a SpringerOpen ${ }^{\odot}$} journal and benefit from:

- Convenient online submission

- Rigorous peer review

- Immediate publication on acceptance

- Open access: articles freely available online

- High visibility within the field

- Retaining the copyright to your article

Submit your next manuscript at $\gg$ springeropen.com 